UCRL-JC-131357

\title{
Preprint
}

\section{Thomson Scattering from Laser Plasmas}

\author{
S. H. Glenzer, W. E. Alley, K. G. Estabrook, J. S. De Groot,
} M. Haines J. H. Hammer, J.-P. Jadaud, B. J. MacGowan, J. D. Moody, W. Rozmus L. J. Suter and E. A. Williams

This paper was prepared for submittal to $40^{\text {th }}$ Annual Meeting of the Division of Plasma Physics

New Orleans, LA

November 16-20, 1998

January 12, 1999

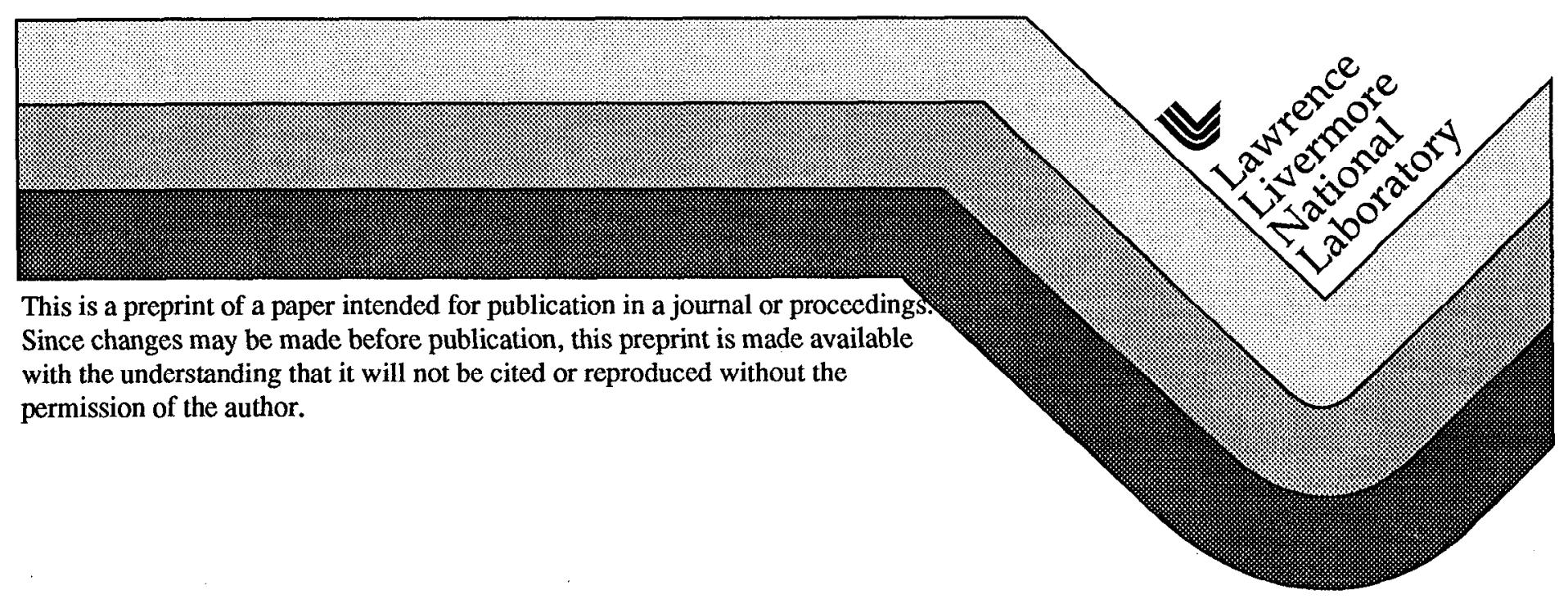




\section{DISCLAIMER}

This document was prepared as an account of work sponsored by an agency of the United States Government. Neither the United States Government nor the University of Califormia nor any of their employees, makes any warranty, express or implied, or assumes any legal liability or responsibility for the accuracy, completeness, or usefulness of any information, apparatus, product, or process disclosed, or represents that its use would not infringe privately owned rights. Reference herein to any specific commercial product, process, or service by trade name, trademark, manufacturer, or otherwise, does not necessarily conslitute or imply its endorsement, recommendation, or favoring by the United States Government or the University of California. The views and opinions of authors expressed herein do not necessarily state or reflect those of the United States Government or the University of California, and shall not be used for advertising or product endorsement purposes. 


\title{
Thomson Scattering from Laser Plasmas
}

S. H. Glenzer, W. E. Alley, K. G. Estabrook, J. S. De Groot*, M. Haines**, J. H. Hammer, J.-P. Jadaúd ${ }^{* * *}$, B. J. MacGowan, J. D. Moody, W. Rozmus ${ }^{* * * *}$, L. J. Suter, and E. A. Williams

L-399, Lawrence Livermore National Laboratory, University of California P. O. Box 808, Ca 94551, U.S.A.

* Department of Applied Science and Plasma Research Group, University of California, Davis, Ca 95616, U.S.A.

** Impcrial Collegc, London SW7 2BZ, United Kingdom

*** CEA/DAM-lle de France, BP12 91680 Bruyères-le-châtel, France.

**** Department of Physics, University of Alberta, Edmonton, Al, Canada T6G2J1 and Institute for Lasers Science Applications, University of California, Ca 94550, U.S.A.

\begin{abstract}
Thomson scattering has recently been introduced as a fundamental diagnostic of plasma conditions and basic physical processes in dense, inertial confinement fusion plasmas. Experiments at the Nova laser facility [E. M. Campbell et al., Laser Part. Beams 9, 209 (1991)] have demonstrated accurate temporally and spatially resolved characterization of densities, electron temperatures, and average ionization levels by simultaneously observing Thomson scattered light from ion acoustic and electron plasma (Langmuir) fluctuations. In addition, observations of fast and slow ion acoustic waves in two-ion species plasmas have also allowed an independent measurement of the ion temperature. These results have motivated the application of Thomson scattering in closed-geometry inertial confinement fusion hohlraums to benchmark integrated radiation-hydrodynamic modeling of fusion plasmas. For this purpose a
\end{abstract}


high energy $4 \omega$ probe laser was implemented recently allowing ultraviolet Thomson scattering at various locations in high-density gas-filled hohlraum plasmas. In particular, the observation of steep electron temperature gradients indicates that electron thermal transport is inhibited in these gas-filled hohlraums. Hydrodynamic calculations which include an exact treatment of large-scale magnetic fields are in agreement with these findings. Moreover, the Thomson scattering data clearly indicate axial stagnation in these hohlraums by showing a fast rise of the ion temperature. Its timing is in good agreement with calculations indicating that the stagnating plasma will not deteriorate the implosion of the fusion capsules in ignition experiments.

PACS numbers: $52.35 \mathrm{Fp}, 52.35 \mathrm{Mw}, 52.40 \mathrm{Nk}, 52.50 \mathrm{Jm}$ 


\section{INTRODUCTION}

Thomson scattering (TS $)^{1-6}$ is a powerful technique for plasma characterization that has seen limited applications in inertial confinement fusion (ICF) plasmas. This is because the small scale, strong laser absorption, stray light, plasma gradients, and multiple ion species in the higher density plasmas relevant to ICF present a significant experimental challenge. Over the last years we have developed TS capability at the Nova laser facility ${ }^{7}$ and we have performed successful TS experiments in ICF plasmas which became feasible due to recent experimental and technical progress in this field. Among these are the verification of the TS theory for plasmas with multiple ion species ${ }^{8}$ and with steep gradients ${ }^{9}$ using open geometry blow-off plasmas as well as the application of high-energy ${ }^{10}$ or ultraviolet ${ }^{11}$ probe lasers. In this paper we will describe that capability and present examples illustrating the importance of the high precision measurements in refining our understanding and ability to calculate laser plasmas.

Our experiments have characterized closed-geometry gas-filled hohlraums allowing detailed comparisons with integrated radiation-hydrodynamic modeling ${ }^{12,13}$ of laser plasmas. Hohlraums are used in indirect drive ICF research ${ }^{14,15}$ to convert shaped high-energy laser light into soft $\mathrm{x}$ rays driving the implosion of the fusion capsule by $\mathrm{x}$-ray ablation pressure. In present ignition design for the future National Ignition Facility (NIF), the hohlraums are filled with a low $-Z$ gas $^{15-17}$ to inhibit movement of the laser absorption and x-ray emission region and to prevent early axial stagnation of the high $-Z$ plasma blow off. These are important properties of gas-filled hohlraums for maintaining radiation symmetry and a symmetric high-convergence, high-yield implosion of the fusion capsule. The TS measurements in hohlraums have allowed us to test the plasma characteristics of this design and are important to understand laser-plasma interactions in gas-filled hohlraums ${ }^{18-23}$. In addition, the TS experiments have changed the way we model laser plasmas.

It was found previously ${ }^{10}$ that the electron temperature measurements were only consistent with radiation-hydrodynamic modeling if a high degree of electron thermal transport 
inhibition was postulated. In the present study, we have verified this finding by performing new ultraviolet $(4 \omega)$ TS experiments in gas-filled hohlraums which are heated with smoothed laser beams. They show high electron temperatures of up to $4.5 \mathrm{keV}$ associated with steep electron temperature gradients. For this reason we have performed the first simulations of hohlraum plasmas that include all relevant large-scale magnetic field terms (see Ref. ${ }^{24}$ ). They show excellent agreement with the experiments without assumptions about heat transport inhibition. These simulations also provide good agreement with our earlier $2 \omega$ TS studies ${ }^{10}$ and verify its conclusions indicating that magnetic fields of order of $\leq 1 \mathrm{MG}$ may be an important source for the inhibition of electron thermal transport ${ }^{25,26}$.

Measurements of the ion temperature in two ion species plasmas have been validated by our experiments with open geometry laser-produced plasmas. In hohlraums that are filled with a carbon-hydrogen gas we find that the ion temperatures increase rapidly on axis during the heating with a shaped laser pulse. The measured timing is in good agreement with the radiation-hydrodynamic modeling which shows on-axis stagnation due to fast compression of the gas plasma. We have investigated this effect for different densities and we find that stagnation is delayed with increasing density as predicted by the modeling. This is an encouraging finding for future indirect drive ignition experiments as it indicates that the high pressure stagnating plasma regions do not affect the capsule implosion for high gas-fill densities.

The paper is organized as follows: In Section IIA we describe the measurement capability, the theory used to analyze the experimental data, and briefly discuss an open-geometry experiment in which detailed measurements of the averaged charge number of Au has been made with TS. These measurements have allowed us to understand the significance of dielectronic recombination to model recombining high $-\mathrm{Z}$ plasmas which is an important test of atomic physics codes used in the modeling of hohlraum plasmas. In Section IIB we will briefly describe an open-geometry experiment which has verified the theory from Fejer ${ }^{27}$ and Evans ${ }^{28}$ for the TS spectra from two-ion species plasmas. This theory is subsequently applied to analyze the TS data from hohlraums. In Section IIIA we present the hohlraum 
experiments and discuss the comparison of the experimental electron temperatures with the radiation-hydrodynamic modeling. The discussion includes the first hohlraum simulations with an exact treatment of large-scale magnetic fields which show good agreement with the TS data. In Section IIIB we describe the modeling with magnetic fields, and in Section IIIC we present a comparison of the experimental ion temperatures with the simulations. Section IV summarizes the results.

\title{
II. VERIFICATION OF THOMSON SCATTERING THEORY WITH OPEN- GEOMETRY EXPERIMENTS
}

\author{
IIA. High-Z Plasmas
}

Shortly after the first application of TS to measure electron densities and temperatures in laboratory discharge plasmas 35 years ago ${ }^{29,30}$, it was established world-wide as a standard technique to characterize low-density magnetic fusion plasmas ${ }^{31,32}$. Subsequently, the utility of TS was also demonstrated in laser-produced plasmas to measure electron temperatures and the characteristics of ion acoustic and electron plasma waves in a large number of studies using open-geometry disk and exploding foil plasmas (e.g., Refs. ${ }^{33-40}$ ). In experiments at the Nova laser facility we have verified the theoretical TS cross section calculations ${ }^{6,27,28}$ for plasmas with steep gradients and with two-ion species using blow-off coronal plasmas in order to understand TS spectra from hohlraums and to correctly deduce temperatures and flow from the experimental data. In particular, we have measured the electron plasma wave spectrum (electron feature) in addition to the ion acoustic wave spectrum (ion feature) from a highly ionized Au plasma with $\mathrm{TS}^{9}$. For similar conditions we have also observed two ion acoustic waves in two ion species plasmas and verified the theoretical predictions for the phase velocities of the waves for various ion concentrations ${ }^{8}$. These studies have shown that electron temperatures, $T_{e}$, electron densities, $n_{e}$, the averaged ionization stage, $\bar{Z}$, ion temperatures, $T_{i}$, and relative ion concentrations of the plasma can be measured with high 
accuracy with error bars of $<20 \%$.

The experiments were performed at the $\mathrm{Nd}$ :glass Nova laser facility at the Lawrence Livermore National Laboratory that operates at $1.055 \mu \mathrm{m}(1 \omega)$ and which can be frequency converted to $2 \omega$ or $3 \omega$. In addition, an independent $4 \omega(50 \mathrm{~J})$ probe laser was recently implemented for TS experiments ${ }^{11}$. To produce a two-ion species plasma with a controlled amount of a light and a heavy species, a flat disk of $2 \mathrm{~mm}$ diameter was illuminated with a single Nova beam (Fig. 1). It is a f/4.3 laser beam illuminating the disk at an angle of $64^{\circ}$ to normal. We used a $1 \mathrm{~ns}$ square pulse and $2.9 \mathrm{~kJ}$ energy at $3 \omega(\lambda=351 \mathrm{~nm})$ resulting in an intensity of $I \cong 10^{15} \mathrm{~W} \mathrm{~cm}^{-2}$ on target. The disks were coated with Au and Be multilayers of varying thickness. For example, we used 860 layers of $0.5 \mathrm{~nm} \mathrm{Au}$ and $5.6 \mathrm{~nm} \mathrm{Be}$ with a total thickness of $2.6 \mu \mathrm{m}$ on a $51 \mu \mathrm{m}$ thick Au or $254 \mu \mathrm{m}$ thick Be substrate to obtain a plasma consisting of $4 \% \mathrm{Au}$ and $96 \% \mathrm{Be}$ by atomic number. For the measurements with pure Au we applied a somewhat longer heater pulse of $1.5 \mathrm{~ns}$ with higher energy and a kinoform phase plate $(\mathrm{KPP})^{41}$ keeping the intensity at $I \cong 10^{15} \mathrm{~W} \mathrm{~cm}^{-2}$.

Initially, TS has been performed with one beam of the Nova laser facility which was operated at $2 \omega(526.6 \mathrm{~nm})$ and at energies of $100-500 \mathrm{~J}$. Recent experiments have characterized the blow-off plasmas applying $4 \omega(263.3 \mathrm{~nm})$ TS at various positions including the high density plasma regions close to the surface of the disk which can not be diagnosed with a $2 \omega$ laser. The $4 \omega$ probe was operated at low energies of $50 \mathrm{~J}$ at $4 \omega$ in a $1.5 \mathrm{~ns}$ square pulse to characterize the coronal temperature and flow gradients of the plasma which is critical to validate the theory used to calculate Thomson scattering spectra. These measurements are described in more detail in Ref. ${ }^{9}$. They show an electron temperature gradient with a scale length of $L_{T}=800-1800 \mu \mathrm{m}$ while the disk is being heated by the $3 \omega$ heater beam. At the end of the heating the corona quickly becomes isothermal. The flow gradients were found to be in the range of $L_{V}=400-800 \mu \mathrm{m}$.

For our TS experiments, the electron densities and temperatures, probe laser wavelengths, and scattering angle result in collective TS from fluctuations characterized by wavenumbers, $k$, such that $\alpha=1 / k \lambda_{D}>2$, where $\lambda_{D}$ is the electron Debye length. In this 
regime, the scattered light spectrum consists of the ion and the electron feature. They correspond to scattering resonances at the ion acoustic wave (iaw) and at the electron plasma wave $(e p w)$ frequencies shifted from the incident probe laser frequency on either side on the frequency scale (redshift and blueshift for copropagating and counterpropagating waves along the scattering vector $\mathrm{k}$ ).

Figure 2 shows TS data at the iaw and epw wave frequencies from a Au coronal plasma using a $2 \omega$ probe laser. For our conditions it was advantageous to use a $\sim 500 \mathrm{~J} 2 \omega$ probe for the obscrvation of the clectron feature because of smaller background levels in the visible spectral range, absence of stray light from the heater beam, and because of the larger peak intensity of the electron feature for larger values of $\alpha$ obtained with a $2 \omega$ probe laser. The beam was parallel to the disk at a distance of $z=450 \mu \mathrm{m}$ and focused to $170 \mu \mathrm{m}$ diameter. In addition to a high resolution $1 \mathrm{~m}$-spectrometer which was used to measure the ion feature employing an S-20 streak camera with a temporal resolution of 30 ps and a wavelength resolution of $0.05 \mathrm{~nm}$, we observed the electron feature in the wavelength range of $550 \mathrm{~nm}$ - $800 \mathrm{~nm}$ using a beam splitter and a 1/4-m spectrometer. A second optical streak camera (S-1) was employed to record the electron feature with a temporal resolution of 150 ps and a wavelength resolution of $1.3 \mathrm{~nm}$. Radiation with wavelengths $\lambda<550 \mathrm{~nm}$ was rejected by an optical filter (BG-550, Schott) to prevent saturation of the detector by the ion feature of the TS spectrum. Both spectrometers observed essentially the same volume in the plasma.

The frequency separation of the two peaks of the ion feature (Fig. 2) is twice the ion acoustic frequency, $\omega_{i a}$,

$$
\left(\frac{\omega_{i a}}{k_{i a}}\right)^{2} \simeq \frac{T_{e}}{M}\left(\frac{\bar{Z}}{1+k_{i a}^{2} \lambda_{D}^{2}}+\Gamma_{i} \frac{T_{i}}{T_{e}}\right) \approx \frac{T_{e}}{M}\left(\frac{\bar{Z}}{1+k_{i a}^{2} \lambda_{D}^{2}}\right)
$$

where $M$ is the ion mass, $T_{i}$ is the ion temperature, $\Gamma_{i}$ is the specific heat ratio, and the length of the scattering vector $k_{i a}$ is given by the triangle relation ${ }^{1}$ shown in Fig. 1. The propagation direction of the corresponding ion acoustic waves in the plasma is determined by the scattering vector $\mathbf{k}$ with $\mathbf{k}=\mathbf{k}_{\mathbf{i a}}=\mathbf{k}_{\mathbf{s}}-\mathbf{k}_{\mathbf{0}}$. The wavenumber of the incident light, $\mathbf{k}_{\mathbf{0}}$, is in the direction of the probe laser. Similarly $\mathbf{k}_{\mathbf{s}}$ points in the direction of the detector. 
More generally, we have

$\mathrm{k}=-\mathrm{k}_{0}+\mathbf{e}_{\mathrm{s}} \frac{\omega+\omega_{0}}{c}$

where $\omega_{0}$ is the frequency of the incident laser and $\omega+\omega_{0}$ is the frequency of the scattered radiation. The incident wave vector must be found from the dispersion relation for electromagnetic waves in plasmas

$\omega_{0}^{2}=\omega_{p}^{2}+k_{0}^{2} c^{2}$

$\mathrm{c}$ is the velocity of light, and $\omega_{p}$ is the plasma frequency. Equation (3) can be solved for $k_{0}$ in terms of the critical density which is defined by $\omega_{0}=\omega_{p}$ and given in practical units as

$\left.n_{c r}\left(\mathrm{~cm}^{-3}\right)=1.1 \times 10^{21} /\left(\lambda_{0}(\mu \mathrm{m})\right)^{2}\right)$

Solving Eq. (3) for $k_{0}$ and using Eq. (4) gives

$k_{0}=\frac{\omega_{0}}{c} \sqrt{1-\left(\frac{\omega_{p}}{\omega_{0}}\right)^{2}}=\frac{\omega_{0}}{c} \sqrt{1-\frac{n_{e}}{n_{c r}}}$

This relation shows that $k_{0}$ is reduced from its vacuum value for high density plasmas. For example, for $n_{e}=2 \times 10^{20} \mathrm{~cm}^{-3}$ and a probe laser with $\lambda_{0}=.5266 \mu \mathrm{m}$ we have $k_{0}=0.97 \frac{w_{0}}{\mathrm{c}}$. This results into a small density-dependent correction. Larger corrections of up to $15 \%$ occur in hohlraums where $n_{e}>10^{21} \mathrm{~cm}^{-3}$. Since the frequency of the scattered light is only shifted by the comparably small ion acoustic frequency, the wavenumber of the scattered light is given by $k_{s} \cong k_{0}$. It is then possible to calculate the wavenumber of the ion acoustic waves from the triangle relation ${ }^{1}$ shown in Fig. 1

$k_{i a} \cong 2 k_{0} \sin (\theta / 2)$

using $k_{0}$ as given by Equation (5). For large frequency shifts of the scattered light as, e.g, for the $e p w$ resonance, the $\mathbf{k}$-vector is given by Eq. (2) with the frequency $\omega$ or the wavelength $\lambda=2 \pi c /\left(\omega+\omega_{0}\right)$ as independent variable.

For example, in case of a highly ionized coronal plasma where the electron temperature exceeds the ion temperature $T_{i}$ so that the $T_{i} / T_{e}$-term in Eq. (1) is negligible, this equation 
illustrates that $T_{e}$ can be deduced from the frequency separation $\omega_{i a}$ if $\bar{Z}$ is known independently or vice versa. However, a rigorous treatment to infer the plasma parameters from the experimental spectra involves fitting of the experimental spectra with the theoretical form factor $S(\mathrm{k}, \omega)^{42-45}$. The theoretical fits shown in Figure 2 use the following expression ${ }^{6}$ for the scattered power, $P_{s}$, into a solid angle $d \Omega$ per frequency range $d \omega$,

$$
\frac{d P_{s}}{d \Omega d \omega}=\frac{\hat{\eta}_{1} \cdot \hat{\eta}_{2}}{2 \pi} r_{0}^{2} \int d x S(\mathbf{k}, \omega ; x) n_{e}(x) \int d y d z \frac{c E_{0}^{2}(\mathbf{r})}{8 \pi}
$$

where $\hat{\eta}_{i}(i=1,2)$ are polarization vectors defining the directions of the Thomson probe electric field $E_{0}$ and of the scattered light detection; $r_{0}=e^{2} / m_{e} c^{2}$. The dynamical form factor, $S\left(\mathrm{k}^{-} \omega ; x\right)$, has been calculated locally to account for spatial variations of electron density $n_{e}(x)$, electron temperature, $T_{e}(x)$ and plasma flow velocity, $V(x)$. We have assumed the following linear forms of these variations: $T_{e}(x)=T_{e}\left(1-x / L_{T}\right), n_{e}(x)=n_{e}\left(1-x / L_{n}\right)$, $V(x)=V\left(1+x / L_{V}\right)$, within the scattering volume which is defined by the geometry of the collection optics and the Thomson probe intensity, $E_{0}^{2}(\mathbf{r})$. Gradients in $V$ and $T_{e}$ primarily affect the iaw spectrum and gradients in $n_{e}$ primarily affect the epw spectrum. The TS cross-section (2) has been derived in the approximation which exploits an existing scale separation between plasma parameter gradients and the characteristic electron fluctuation wavelength, i.e. for $k L_{n}, k L_{T}, k L_{V} \gg 1$. The quantities $L_{T}, L_{V}$ have been inferred from $4 \omega$ TS data which have been collected at different locations along the normal direction to the disk.

The inhomogeneity of a plasma flow velocity $V(x)$ is primarily responsible for the large broadening of ion acoustic resonances shown in Fig. 2. For comparison, we also show in Fig. 2 a calculated iaw spectrum without including gradients (dashed curve) which clearly underestimates the width of the resonance. Other small sources of broadening of the iaw resonance are ion-ion collisions ${ }^{46}$ and electron Landau damping in these high- $Z$ plasmas. A collisionless response of electrons is justified by the large values of the ratio between electron-ion mean free path and the wavelength of fluctuations, $k \lambda_{e i} \gg 1$. An expression for the dynamical form factor, $S(\mathbf{k}, \omega)$, which accounts for the effect of ion-ion collisions has 
been derived in Ref. ${ }^{47}$ from a theory of hydrodynamical fluctuations. It has the following form,

$S(k, \omega)=\frac{4 k^{2}\left[c_{s}^{2} /\left(1+k^{2} \lambda_{D}^{2}\right)+v_{i}^{2}\right] \gamma_{i a}}{\left(\omega^{2}-k^{2} v_{s}^{2}\right)^{2}+4 \omega^{2} \gamma_{i a}^{2}}$

where $v_{s}=\left[c_{s}^{2} /\left(1+k^{2} \lambda_{D}^{2}\right)+\Gamma_{i} v_{i}^{2}\right]^{1 / 2}, c_{s}=\left(\bar{Z} T_{e} / M\right)^{1 / 2}, v_{i}=\left(T_{i} / M\right)^{1 / 2}$. The damping of ion-acoustic fluctuations at the frequency $\omega, \gamma_{i a}=2 k^{2} v_{i}^{2} R e \eta_{i} / 3 \nu_{i}+(\pi / 8)^{1 / 2} c_{s} k v_{s} / v_{e}$ and the specific heat ratio, $\Gamma_{i}=5 / 3+4 \omega I m \eta_{i} / 3 \nu_{i}$, are defined by the frequency dependent ion $\operatorname{viscosity}^{47}, \eta_{i}=i \nu_{i}\left(\omega+i 1.46 \nu_{i}\right) /\left[\left(\omega+1.2 i \nu_{i}\right)\left(\omega+1.46 i \nu_{i}\right)+0.23 \nu_{i}^{2}\right]$. Ion-ion collisions are described by the frequency $\nu_{i}=4 \sqrt{\pi} \bar{Z}^{4} e^{4} n_{i} \Lambda_{i} / 3 M^{1 / 2} T_{i}^{3 / 2}$. In addition in order to reproduce the TS data as shown in Fig. 2 we have modified the frequency $\omega$ by a Doppler shift, $\mathbf{k} \cdot \mathbf{V}(\mathbf{x})$ due to projection of a plasma flow velocity on the direction of the wave number $k$.

The simultaneous observation of the $i a w$ and the $e p w$ wave spectrum offers a unique way to obtain $T_{e}, n_{e}$, and $\bar{Z}$ from TS alone. For this purpose we first determine $T_{e}$ and $n_{e}$ from the $e p w$ spectrum and use the value for $T_{e}$ to obtain $\bar{Z}$ from the $i a w$ spectrum. Figure 2 shows a heavily damped epw resonance which wavelength shift is approximated by the Bohm-Gross dispersion relation

$$
\omega^{2}=\omega_{p}^{2}+3 k^{2} T_{e} / m_{e}
$$

where $\omega_{p}^{2}=4 \pi e^{2} n_{e} / m_{e}$ is the plasma frequency. Equation (9) indicates that the electron density of the plasma can be obtained with high accuracy. For this purpose, the electron temperature must be self-consistently obtained from the epw spectrum which is possible as the electron plasma wave is heavily damped by electron Landau damping. The fit of the spectrum includes gradients where we take the electron density gradient from the radiationhydrodynamic modeling. Again, it turns out that the gradients are important only while the heater beam is on at $t=1.4 \mathrm{~ns}$. Without gradients the width of the peak at $735 \mathrm{~nm}$ is smaller than observed (dashed curve in Fig. 2). The values of $L_{n}, L_{V}$ and $L_{T}$ used in the modeling of the $e p w$ resonance are consistent with the inhomogeneity scales applied to the iaw resonance calculations. It turns out that the fit is fairly robust. Increasing 
Landau damping by increasing $T_{e}$ results in a broader electron feature, but also shifts the resonance to larger wavelengths (Bohm-Gross relation). To compensate for this shift, one has to assume a smaller electron density which in turn reduces electron Landau damping, and this fit does not converge. Small deviations from the calculated electron density gradient are probably responsible for the slight discrepancies between the fit and the data at $\lambda \approx 780 \mathrm{~nm}$. Consistent with this picture, discrepancies of this kind are not present for the data taken after the heater beam turns off when gradients are less important (see Ref..$^{9}$ ). To model the TS data at the epw resonance we have used in Eq. (2) a collisionless expression for the dymanical form factor (cf e.g. [4])

$S(\mathrm{k}, \omega)=\frac{2 \pi}{k}\left|1-\frac{\chi_{e}}{\epsilon}\right|^{2} \bar{F}_{0 e}(\omega / k)+\frac{2 \pi \bar{Z}}{k}\left|\frac{\chi_{e}}{\epsilon}\right|^{2} \bar{F}_{0 i}(\omega / k)$

where the plasma dielectric function, $\epsilon=1+\chi_{e}+\chi_{i}$, and the susceptibility functions, $\chi_{\alpha}=1+\left(\omega_{p \alpha}^{2} / k^{2}\right) \int d^{3} v(\omega-\mathbf{k} \cdot \mathbf{v})^{-1} \mathbf{k} \cdot \partial F_{0 \alpha} / \partial \mathbf{v}(\alpha=e, i)$ are defined using the local Maxwellian distribution functions, $F_{0 \alpha}$. The symbol $\bar{F}_{0 \alpha}$ stands for a one dimensional distribution function which has been obtained after integration of the components of the velocity vector transverse to $\mathbf{k}$. The distribution functions depend on the spatial coordinate $x$ through inhomogeneous flow velocity and electron temperature. In addition, by fitting the spectrum we can exclude the possible presence of non-Maxwellian distributions ${ }^{48-50}$ in the plasma. Predictions from Fokker-Planck calculations ${ }^{51}$ result in a super-Gaussian distribution, $F_{0 e} \sim \exp \left(-v^{m}\right)$, with an exponent in the range of $m=3.5-4$ for the averaged intensity of the $2 \omega$ probe laser. However, we find by replacing $F_{0 c}$ in Eq. (10) with the superGaussian distribution ${ }^{49,48}$ that the electron feature is fit best by a Maxwellian distribution $(m=2)$. while, e.g., $m=3$ results in a resonance that is too narrow, not consistent with the gradients in the plasma. The predictions likely overestimate $m$ because in the present experiment, lateral electron transport out of the probe beam volume plays an important role which is not accounted for in the calculations. The fit shown in Fig. 2 gives $T_{e}=2.0 \mathrm{keV}$ and $n_{e}=2.1 \times 10^{20} \mathrm{~cm}^{-3}$.

Using the parameters from the epw resonance, we obtain $\bar{Z}$ of the plasma by fitting the 
simultaneously measured ion acoustic wave spectrum. The ion acoustic peaks are significantly broadened due to the inhomogeneity of the plasma in the scattering volume (Fig. 2). The measured temperature gradient implies a heat flux and a corresponding skewing of the distribution function ${ }^{50}$. This results in different Landau damping of the two ion acoustic peaks. Accounting for this asymmetry, the broadening from the instrument $(0.05 \mathrm{~nm})$, and the spatial gradients allows a good fit of the entire spectrum. $T_{e}$ is given by the electron feature with an error of about $15 \%$, by varying the calculated spectra within the noise of the data. Therefore, we can deduce the averaged charge state $\bar{Z}$ from the ion feature with an error of $20 \%$.

As an example, we show in Fig. 3 the averaged charge number $\bar{Z}$ measured with TS together with two-dimensional simulations using the radiation-hydrodynamic code LASNEX. Although in this study we can generally neglect heating of the $4 \omega$ probe laser, the high-energy $2 \omega$ probe must be included in the simulations. These assumptions were verified by varying the energy of probe lasers over three orders of magnitude. The good agreement between the measured and calculated electron temperatures suggests that the simulations approximate well the heating of the probe laser as well as the electron thermal transport in the corona plasma. The experimental data show that the charge number decreases from $\bar{Z}=(45 \pm 9)$ to $\bar{Z}=(25 \pm 5)$. Both the calculations based on an average atomic physics model $(\mathrm{XSN})^{52}$ and the calculations with detailed configuration accounting (DCA, $\sim 1000$ levels $)^{53}$ agree with the measurements during the heater beam pulse giving $\bar{Z} \simeq 49$. After the heater has turned off, we observe that the averaged atomic physics model significantly overestimates the charge state of the plasma. On the other hand, the DCA model is in reasonable agreement with the experimental data. DCA calculations without two electron transitions processes show that dielectronic recombination is the process responsible for the decrease of $\bar{Z}$ of the plasma as seen in the experiment. Consistent with this observation of a recombining plasma, we find that the simultaneously measured electron density also falls rapidly. Independent from these findings we have verified the importance of dielectronic recombination for the present conditions with Monte Carlo calculations ${ }^{54}$. Furthermore, 
recent measurements have observed a fast decrease of $\bar{Z}$ with $\mathrm{x}$-ray emission spectroscopy for the same experimental conditions.

\section{IIB. Two-Ion Species Plasmas}

Figure 4 shows the TS data recorded from a $4 \% \mathrm{Au}, 96 \%$ Be disk target $\left(\right.$ from Ref. $^{8}$ ). Four ion acoustic features, the two outer ones belonging to $\mathrm{Be}$ and the two inner ones belonging to $\mathrm{Au}$, can be clearly seen for $0.9 \mathrm{~ns}<t<1.7 \mathrm{~ns}$. This finding verfies theoretical predictions of Fejer ${ }^{27}$ and Evans ${ }^{28}$. In Fig. 4 the $3 \omega$ heater beam lasts from 0 to 1 ns and the $2 \omega$ probe beam from 0 to 4 ns. We find that the TS spectra are Doppler shifted to the blue due to the supersonic expansion of the corona. This can easily be seen from the $2 \omega$ stray light which can be used as a convenient wavelength and timing fiducial. For the time interval shown in Fig. 4(a), Thomson scattering gives temperatures of the plasma of $0.2 \mathrm{keV}<T_{e}<1.2 \mathrm{keV}$ ensuring that the Be ions are fully ionized. Therefore, the separation and damping of the ion acoustic features belonging to the $\mathrm{Be}$ ions can be directly used to determine the electron temperature. Peak electron temperatures are smaller than in the previously described experiments because the low $-\mathrm{Z}$ species dominate the composition of the plasma giving rise to a more isothermal corona. In addition, the heater beam is shorter. The electron densities are of order $\sim 10^{20} \mathrm{~cm}^{-3}$ giving a scattering parameter $\alpha$ well above 3 in which case scattering spectra are not sensitive to the electron density.

The TS spectrum at $1.5 \mathrm{~ns}<t<1.6 \mathrm{~ns}$ shows the four ion acoustic resonances due to the slow and the fast ion acoustic wave [Fig. 4(b)]. Apart from the slight asymmetry, analysis of the spectrum yields considerable information. Since the relative intensities of the ion acoustic peaks are determined by damping of the $\mathrm{Au}$ and $\mathrm{Be}$ ion acoustic waves, the theoretical fit to the spectrum gives $T_{i} / T_{e}=1.1[\mathrm{Fig} .4(\mathrm{c})]$. For the spectrum shown in Fi. 4(b) gradients are not important since it is taken after the heater beam turns off and in addition because low $-\mathrm{Z}$ blow-off plasmas tend to be isothermal ${ }^{55}$. From the wavelength separation of the Be ion acoustic features, we find $T_{e}=230 \mathrm{eV}$. Finally, the wavelength 
separation of the $A u$ features gives $\bar{Z}=40$. We have performed this analysis to a series of experiments with relative $\mathrm{Au}$ concentrations of $1 \%, 2 \%, 4 \%$, and $8 \%$. The ratio of the wavelength separation of the $\mathrm{Au}$ ion acoustic features to the $\mathrm{Be}$ ion acoustic features was found to increase with increasing Au concentrations (see Ref. ${ }^{8}$ ) providing a detailed verification of the theory for $S(\mathbf{k}, \omega)$ of Fejer $^{27}$ and Evans ${ }^{28}$.

Our data show that Thomson scattering from a two ion species plasma is an accurate diagnostic of electron temperature and ion temperature. The observation of both the fast and the slow wave on the Thomson scattering spectra shows that they are moderately to strongly damped ${ }^{56}$. In this regime, Landau damping is a strong function of the ion temperature resulting in a highly accurate ion temperature measurement. In addition, the relative ion densities can be measured with high accuracy if the ion charge state is known independently or vice versa.

\section{HOHLRAUM PLASMAS}

IIIA. Experiments with Gas-filled Hohlraums

Figure 5 shows the experimental geometry of a hohlraum TS experiment. Cylindrical gold hohlraums of $2,750 \mu \mathrm{m}$ length and $800 \mu \mathrm{m}$ radius (scale-1) were used which is the standard size for capsule implosions $s^{57,58}$ and present benchmarking experiments ${ }^{10,23,59,60}$ at Nova. On either side, five laser beams enter the hohlraum through laser entrance holes (LEH). We shot hohlraums filled with 1 atm of methane $\left(\mathrm{CH}_{4}\right)$ or with 1 atm of propane $\left(\mathrm{C}_{3} \mathrm{H}_{8}\right)$ and used $0.35 / \mathrm{m}$ thick polyimide to cover holes. The hohlraums were heated with a total of ten KPP-smoothed laser beams. The pulse duration was $2.4 \mathrm{~ns}$ rising from a $7 \mathrm{TW}$ foot to 17 TW peak power (pulse shape no. 22: PS22) with a total energy supplied to the target of $27 \mathrm{~kJ}$.

We used the $4 \omega$ probe laser to measure TS spectra from various locations in the hohlraim. The probe was focused to $\sim 100 \mu \mathrm{m}$ diameter giving a cylindrical scattering volume. Its 
length is determined by the imaging optics $(f / 10)$ and is $70 \mu \mathrm{m}$ for the presently used 1.5 magnification and $100 \mu \mathrm{m}$ detector slit size. The scattering angle was chosen to be $\theta=90^{\circ}$ and the scattered light was observed through a rectangular window $(400 \mu \mathrm{m} \times 600 \mu \mathrm{m}) \mathrm{cut}$ in the side of the hohlraum.

Figure 6 shows the $4 \omega$ TS data from the axis of a methane-filled hohlraum at a distance of $800 \mu \mathrm{m}$ from the hohlraum center. For $0 \mathrm{~ns}<t<1.1$ ns no TS signal can be observed. Estimates show that the TS signal is not detectable because of the small electron density in the scattering volume at early times. For $1.1 \mathrm{~ns}<t<1.8 \mathrm{~ns}$ two broad ion acoustic features are observed from light scattering off the $\mathrm{CH}_{4}$-plasma. The intensity and the width of the ion acoustic peaks increases significantly during this period indicating increasing densities and ion temperatures. Furthermore, the TS spectrum shows an asymmetry consistent with an electron heat flux from the LEH region, where all the heater beams cross, to the center of the hohlraum. The heat flow increases the electron Landau damping of the ion acoustic wave resonance in the direction of the flow ${ }^{61,62}$ which is the red component of the TS spectrum of Fig. 6. In Fig. 6 we also observe a small red shift of the whole TS spectrum. This wavelength shift gives information about macroscopic plasma flow in hohlraums which is an important parameter affecting laser beam pointing ${ }^{63}$. It indicates a plasma motion from the LEH towards the hohlraum center with a speed of $v \simeq 1.3 \times 10^{7} \mathrm{~cm} / \mathrm{s}$ which is of the same order as seen in the simulations $\left(v=2 \times 10^{7} \mathrm{~cm} / \mathrm{s}\right)$. For $t>1.8 \mathrm{~ns}$, a cut-off of the Thomson scattering signal occurs because the probe laser is absorbed when the high density Au plasma from the hohlraum walls moves into the path of the probe laser.

Fitting the hohlraum TS data with Eq. (7) using the form factor $S(\mathbf{k}, \omega)$ for a two ion species plasma, which has been tested with experiments described above, gives accurate data for the electron and ion temperature of the plasma. Figure 6(b) shows the spectra at $t=1.2 \mathrm{~ns}$ and $t=1.6 \mathrm{~ns}$ averaged over 80 ps together with the fit. For the data analysis it is assumed that light scattering occurs on a fully ionized $\mathrm{CH}$-plasma with $T_{C}=T_{H}$ because of equilibration times of order 0.2 ns. Our hydrodynamic simulations as well as temporally resolved two-dimensional x-ray images observing the Au-plasma emission at energies of $E>$ 
$2.5 \mathrm{keV}$ show that Au-ions were not present in the scattering volume for $t<1.8 \mathrm{~ns}$ (Fig. 7). The x-ray images view the hohlraum plasma along its axis. They show a five-fold radiation pattern due to the gold blowing off the wall where the heater beams illuminate the hohlraum. From Fig. 7 it is obvious that there are no large amounts of Au-ions present on the axis of a gas-filled hohlraum during the time of observation. Calculations show that undetectable small amounts of gold of the order of $1 \%$ have no effect on the measured spectra.

Figure 8 shows the axial electron temperatures inferred from the TS spectra measured at $t=1.3 \mathrm{~ns}$ and at various distances from the hohlraum center together with the results of the radiation-hydrodynamic LASNEX simulations. The results for $T_{i}$ are discussed in Sec. IIIC. The experimental electron temperature data clearly show a steep gradient from about $2 \mathrm{keV}$ closer to the hohlraum center to up to $4.5 \mathrm{keV}$ at a distance of 300 microns from the laser entrance holes where the heater beams cross on their way to the hohlraum wall. The error bars are obtained by varying the fits within the noise of the experimental data and in addition by varying the electron temperature gradient in Eq. (7). Although gradients primarily affect the measurement of the ion temperature they also result in a small uncertainty in $T_{e}$ of about $5 \%$. The total error for $T_{e}$ is typically about $15 \%$. The gradients also affect the symmetry of the TS spectra, and in this study we obtain a qualitative understanding of the direction of the heat flow from a symmetry analysis. For a more quantitative understanding of heat transport it will be necessary to quantify currents in the stagnating plasma and to include non-local electron thermal transport ${ }^{64}$ in calculations of $S(\mathbf{k}, \omega)$.

For the data analysis we have calculated the scattering parameter $\alpha$ using electron densities from $\mathrm{LASNEX}^{13}$. Although for the present experiment we have $\alpha>3$ so that the spectra are not density sensitive, the $\mathbf{k}$ vector was slightly corrected from its vacuum value ${ }^{37}$ since electron densities in the scattering volume approach $0.15 n_{c r}$. We have $k_{i a}=\left(4 \pi / \lambda_{0}\right) \sin (\theta / 2)\left(1-n_{e} / n_{c r}\right)^{1 / 2}$, and $n_{c r}$ is the critical density for the $4 \omega$ probe. Ignoring this effect would result in electron temperatures reduced by $<15 \%$.

In Fig. 8 we compare the experimental data with two-dimensional radiationhydrodynamic simulations where the hohlraum axis is chosen to be the axis of symmetry. 
The calculated temperatures are taken along the axis averaged over $60 \mu \mathrm{m}$ consistent with the size of the scattering volume. This comparison is justified because the probe beam is not affected by beam deflection or refraction. The radiation-hydrodynamic simulations use the code LASNEX with two approximations: The first model uses a flux-limited diffusion model for heat transport where the heat flow per unit area in regions of large classical heat flow $-\kappa \nabla T_{e}$ has an upper bound of $f\left(n_{e} T_{e} v_{e}\right)$, where $n_{e} T_{e} v_{e}$ is the so-called free streaming value of heat transport and $f$ is the flux limit chosen to be $f=0.05$. The second model includes large-scale magnetic fields and is described in some detail in Sec. IIIB.

The comparison in Fig. 8 clearly shows that the inclusion of magnetic fields into the modeling results in excellent agreement between the TS data and the calculations. The Inagnetic fields inhibit the electron thermal transport developing hot regions in the hohlraum (see next chapter). Our modeling of earlier $2 \omega$ TS experiments have used an artificial inhibition of thermal transport by reducing the heat transport flux limiter to $f=0.01$ which, in hohlraums, is cquivalent to reducing classical transport by a factor of ten ${ }^{10}$. Magnetic fields or non-local effects were assumed to cause the heat flow inhibition. The new $4 \omega$ TS data are complementary to our earlier $2 \omega$ TS data from hohlraums which were heated without applying laser beam smoothing techniques. The new data verify our earlier conclusions about heat transport inhibition. In addition, they characterize electron temperature gradients in hohlraums thus providing a detailed data set to benchmark the radiation-hydrodynamic modeling.

\section{IIIB.Comparison with Modeling}

In this Chapter we present calculations which show that the spontaneous generation of magnetic fields at the hohlraum walls and their convection into the low $-Z$ gas plasma affect the thermal transport and therefore the electron temperatures inside the gas plasma through which the heater beams propagate. Early predictions ${ }^{26}$ of magnetic fields in hohlraums resulted in inhibition of electron heat transport by a factor of 20 . Other studies of magnetic 
fields in laser-produced plasmas ${ }^{65}$ have focussed on the mechanisms to generate these fields and their effect on the properties of the plasma neglecting many of the terms that generate or affect magnetic fields. In this study we have performed the first calculations of ICF hohlraum conditions with all relevant magnetic field terms. Of particular interest is the Nernst convection of the magnetic field by the heat flux that can result in local magnification of the field, and the thermomagnetic instability that is dependent on the Righi-Leduc heat transport coupled to the magnetic field generated from the Nernst effect and the electron pressure gradient.

We applied a field solver with a single thermal electron component which is incorporated into the two-dimensional Lagrangian radiation-hydrodynamic code LASNEX ${ }^{12}$. The field solution goes beyond the magnetohydrodynamic (MHD) limit since the full Maxwell field equations are solved, including the displacement current so that charge separation is included. A generalized Ohm's law defines the current density. In the co-moving fluid frame the current density (in Gaussian units) obeys the following equation:

$\frac{\partial \mathbf{J}}{\partial t}+\mathbf{J} \times \Omega=\frac{e}{m_{e}} \nabla P_{e}+\frac{\omega_{p}^{2}}{4 \pi} \mathbf{E}+\mathbf{R}$,

where $\Omega=e \mathrm{~B} / m_{e} c$ is the electron cyclotron frequency, $P_{e}=n_{e} k_{B} T_{e}$ is the electron pressure, $\mathbf{E}$ is the electric field, $\mathbf{R}$ is related to the collisional contribution to the momentum flux that is given by Braginskii ${ }^{25}$ as a constitutive relation relating the drift velocity and the electron temperature gradient to the momentum flux and, hence, the electric current. The terms are broken into components both parallel and perpendicular to the magnetic field with proportionality coefficients relating the resulting current to the driving term. In a slightly different notation than Braginskii $\mathbf{R}$ is written:

$$
\begin{aligned}
\mathbf{R}= & -\left(\nu_{\|}-\nu_{\perp}\right)\left(\mathbf{e}_{\mathbf{b}} \cdot \mathbf{J}\right) \mathbf{e}_{\mathbf{b}}-\nu_{\perp} \mathbf{J}-\nu_{\times}\left(\mathbf{J} \times \mathbf{e}_{\mathbf{b}}\right) \\
& +\left(\beta_{\|}-\beta_{\perp}\right)\left(\mathbf{e}_{\mathbf{b}} \cdot \nabla T_{e}\right) \mathbf{e}_{\mathbf{b}}+\beta_{\perp} \nabla T_{e}+\beta_{\times}\left(\mathbf{e}_{\mathbf{b}} \times \nabla T_{e}\right)
\end{aligned}
$$

where $\mathbf{e}_{\mathbf{b}}$ is the unit vector in direction of the magnetic field, the coefficients $\nu_{i}$ are the collision rates where the subscripts denote parallel, $\|$, perpendicular, $\perp$, and cross, $\times$. The 
cross collisional term with $\nu_{\mathrm{x}}$ is sometimes called the collisional Hall term. The coefficients $\beta_{i}$ multiply the thermal electric terms. The third of the thermal electric terms, with $\beta_{x}$, is often called the Nernst term. The Nernst term plays a role in convection of the magnetic field along a temperature gradient ${ }^{66}$. One term which has not been included is the tensor pressure which is second order in velocity and which therefore adds a small directional perturbation to the pressure. However, since the pressure is taken to be a scalar in Eq. (11), the Weibel instability has been neglected. For hohlraum calculations with cylindrical symmetry and toroidal magnetic fields, Eq. (12) simplifies since the first and fourth term become zero.

The heat conduction equation has been augmented by the inclusion of Braginskii's constitutive relation for the heat flux, here rewritten using a slightly different notation than Braginskii:

$$
\begin{aligned}
\mathbf{Q}= & -\left(\gamma_{\|}-\gamma_{\perp}\right)\left(\mathbf{e}_{\mathbf{b}} \cdot \mathbf{J}\right) \mathbf{e}_{\mathbf{b}}-\gamma_{\perp} \mathbf{J}-\gamma_{\times}\left(\mathbf{e}_{\mathbf{b}} \times \mathbf{J}\right) \\
& +\left(\kappa_{\|}-\kappa_{\perp}\right)\left(\mathbf{e}_{\mathbf{b}} \cdot \nabla T_{e}\right) \mathbf{e}_{\mathbf{b}}-\kappa_{\perp} \nabla T_{e}-\kappa_{\times}\left(\mathbf{e}_{\mathbf{b}} \times \nabla T_{e}\right),
\end{aligned}
$$

where in the limit of zero magnetic field $\gamma_{\perp}$ is related to $\beta_{\perp}$ through an Onsager reciprocal relation. The $\kappa_{i}$ are the thermal conductivities. The term with $\kappa_{\times}$is sometimes called the Righi-Leduc term which plays a key role in the thermomagnetic instability ${ }^{67}$.

The various terms which contribute to the generation of large-scale magnetic fields can be found by solving Eq. (11) for the electric field and substituting into Faraday's law ${ }^{68}$. Some of the terms which result are: the $\nabla T_{e} \times \nabla n_{e}$ source term, magnetic convection, resistive diffusion, magnetic curvature, magnetic pressure, and thermal force terms. These terms are all of the same order and must all be included in the simulations. Magnetic fields produced by hot electrons are not included as well as small-scale fields of the size of laser filaments.

Figure 9 shows the evolution of the toroidal magnetic fields in gas-filled hohlraums calculated with LASNEX using the magnetic field model described above. At early times magnetic fields of the order of $1 \mathrm{MG}$ are produced at the hohlraum walls where the laser beams initially deposit their energy. In addition, the hohlraum window is also magnetized. These fields convect into the low $-Z$ plasma of the hohlraum causing inhibition of electron 
thermal conduction since the product of the electron cyclotron frequency and electron-ion collision time $\omega_{e} \tau_{e}$ approaches values of ten. In particular, in some regions which are traversed by the laser beams (indicated as dotted lines in Fig. 9) the heat tends to be confined for long periods of time comparable to the duration of the hohlraum heating.

Figure 10 demonstrates this effect by showing a two-dimensional contour plot of the electron temperature. Hot plasma regions develop in the path of the laser beams which are surrounded by large magnetic fields. Furthermore, the temperature gradient along the axis of the hohlraum clearly correlates to the presence of the magnetic fields. Our TS measurements have observed both regions of hot plasma, the hot off-axis regions in Ref. ${ }^{10}$, and the axial temperature gradient in Figure 8. The good agreement of this model with the TS data is very encouraging as its demonstrates our level of theoretical understanding of hohlraum plasma conditions and the importance of magnetic fields in ICF hohlraums. These findings will help us to develop our understanding of laser scattering losses by SBS and SRS in hohlraums. A direct effect on present NIF hohlraums designs due to the changed plasma conditions is a slight modification of the optimal beam pointing.

\section{IIIC.Stagnation Physics and Scaling to Higher Densities}

Electron and ion temperatures as a function of time on the axis $(x=800 \mu \mathrm{m})$ of a methane-filled hohlraum are shown in Fig. 11. The ion temperature of the plasma is inferred from the width of the ion acoustic waves giving their damping and therefore the ion temperature. In principle, plasma gradients, turbulence or velocity fluctuations can all affect the results. On the other hand, when an independent determination of the ion temperature is available, the TS spectra provide an estimate of gradients or turbulence in the plasma. A large number of studies using different targets at Nova did not show the presence of turbulence or velocity fluctuations while plasma gradients have been shown to be important (Sec. IIA). Moreover, our experiments with two-ion species plasmas where the ion temperature is inferred from the relative damping of the ion acoustic waves which belong to the 
two ion species and not from the width of the waves give a clear indication of the absence of turbulence in these plasmas. Since in the present study we have measured the electron temperature gradients independently, $T_{i}$ can be inferred with error bars of order $25 \%$ by applying Equation (7).

The ion temperatures are less sensitive to the heat transport model in the simulations since they are determined by $p d V$-work when the low $-Z$ plasma is compressed on the axis by the inblowing gold plasma from the hohlraum walls. The experimental ion temperatures show a fast rise at $t=1.2 \mathrm{~ns}$ to a peak value of $T_{i}=(2.8 \pm 1) \mathrm{keV}$. The hydrodynamic simulations confirm that the rise of $T_{i}$ is due to stagnation of the compressed low- $Z$ plasma on the axis of the hohlraum where $90^{\circ}$ particle scattering occurs on a scale length of $30 \mu \mathrm{m}$. Heating of the ions by shocks or magnetic field compression is negligibly small. In addition, electron-ion temperature equilibration times are too large ( $>1 \mathrm{~ns})$ to explain the experimental data. In Fig. 11 we observe agreement between the experimental and calculated ion temperatures. With exception of the data point at $t=1.2 \mathrm{~ns}$, the measured electron temperatures also agree fairly well with the simulations. The discrepancy for the early time data might be explained by small variations of order $100-200 \mu \mathrm{m}$ of the calculated location of the magnetic fields. The results presented in Fig. 11 indicate that stagnation processes in gas-filled hohlraums are well described by the simulations

This is an important finding because the stagnating plasma generates a significant pressure which can affect the capsule dynamics in ignition experiments. For example, early NIF hohlraum designs used plastic liners ${ }^{15}$ instead of a gas fill to produce the low $-Z$ beam propagation region in the hohlraum interior. These liners blew inward at high velocity and generated an early time axial stagnation pressure which affected the subsequent capsule implosion symmetry. These stagnation features are also observed with temporally-resolved two-dimensional x-ray images of empty gold hohlraums shown in Fig. 7(a). At $t=0.45 \mathrm{~ns}$ the gold blow-off plasma stagnates in between the laser spots and is subsequently filling the hohlraum with a high-density highly radiating plasma [see image at $t=1.8 \mathrm{~ns}$ in Fig. 7(a)]. Gas-filled hohlraums, on the other hand, do not show these emission features because stag- 
nation is weaker and because of the absence of gold on the hohlraum axis. The calculations of (cryogenic) gas-filled hohlraums for the NIF show that the high gas pressures occur late, after the capsule has generated a large surrounding region of ablated material. Because the capsule is moving inward and the radiation ablated capsule blowoff is moving outward at speeds comparable to the sound speed in the gas fill, the capsule is essentially isolated from the pressure generated in the gas. In the present study we have further tested these predictions by performing TS in gas-filled hohlraums at Nova with higher, more NIF-like, gas-fill densities.

Figure 12 shows the experimental $4 \omega$ TS data from a propane-filled $\left(\mathrm{C}_{3} \mathrm{H}_{8}\right)$ hohlraum (a) along with the electron and ion temperatures as a function of time (b). With exception of the gas fill all the other experimental parameters were kept the same as in the methane-filled hohlraum experiments. The characteristics of the streak data shown in Fig. 12(a) are rather similar to the lower density hohlraum results: They show narrow ion acoustics peaks which intensify and broaden during the heating of the hohlraum along with a slight red shift and asymmetry of the TS spectrum. They indicate increasing densities and ion temperatures on the hohlraum axis along with a macroscopic plasma and heat flow from the LEH region towards the hohlraum center. However, the onset of stagnation, measured by the fast rise of $T_{i}$, is delayed by 0.3 ns for the higher-density propane gas fill [Fig. 12(b)].

The delay of the stagnation time is in good agreement with the radiation-hydrodynamic calculations verifying that a higher density gas fill in ignition target designs will be beneficial in preventing early axial stagnation. The fill density will then be limited by the tolerable level of laser scattering losses and the hot electron production by parametric instabilities ${ }^{15,69,70}$. The macroscopic flow seen in the experiments at this location is of the same order as calculated with LASNEX. The comparison of the experimental electron temperatures with the calculations is satisfying while the measured ion temperatures are $50 \%$ larger than calculated. In this high-density case, the comparison is limited because of the fewer number of experiments so that gradients are not as well characterized as in the methane case. In addition, we observe appreciably higher backscatter losses due to SBS and SRS (13\% SBS 
and $9 \%$ SRS for a propane-filled hohlraum compared to $5 \%$ SBS and $0.5 \%$ SRS for a methane-filled hohlraum. Although the losses are taken into account into the modeling by subtracting them from the incident laser power, they introduce an additional uncertainty in the comparison of the absolute values for $T_{e}$ and $T_{i}$.

\section{CONCLUSIONS AND OUTLOOK}

Thomson scattering experiments at Nova have changed the way we model laser plasmas. They have shown the need to take into account heat transport limiting effects such as magnetic fields and to use sophisticated atomic physics radiation packages to model the conditions in ICF plasmas.

We have extensively tested and verified the theory for TS spectra in plasmas with multiple ion species and with steep gradients with open-geometry experiments. The experiments have further demonstrated that TS is a novel experimental technique to measure the temperatures, density, and the averaged charge number $\bar{Z}$ of high $-\mathrm{Z}$ plasmas with high spatial and temporal resolution.

The applications of TS to characterize ICF hohlraum plasmas have pushed the limits of its applicability ${ }^{10,71,72}$ to a parameter range of $1 \mathrm{eV}<T_{e}<5 \mathrm{keV}$ and $10^{11} \mathrm{~cm}^{-3}<n_{e}<$ $3 \times 10^{21} \mathrm{~cm}^{-3}$. We have observed steep electron temperature gradients which are modeled well by radiation-hydrodynamic simulations when including magnetic fields. It will be interesting for future studies to explore TS for the measurements of local magnetic fields (e.g Ref. ${ }^{73}$ ) in a closed-geometry hohlraum plasma to verify the presence of mega-Gauss fields predicted by the simulations. Our measurements of the ion temperature have shown that axial stagnation in these gas-filled hohlraums is well described by the simulations. These results have shown that the gas fill will be an efficient tamper for future ICF experiments.

At present, we are applying TS at Nova to a number of experiments for plasma characterization and to test our understanding of high temperature ICF plasmas. These tests involve studies of non-LTE radiation physics models in high $-\mathrm{Z}$ plasmas, predictions of Mach-1 
flow in exploding foil plasmas, studies of fluctuations, and investigations of the properties of laboratory astrophysical plasmas.

\section{ACKNOWLEDGMENTS}

The authors would like to thank H. A. Baldis, E. M. Campbell, B. A. Hammel, and J. D. Kilkenny for helpful discussions and support. We also thank the Nova crew for their efforts. This work was performed under the auspices of the U.S. Department of Energy by the Lawrence Livermore National Laboratory under contract No. W-7405-ENG-48. 


\section{REFERENCES}

1. H.-J. Kunze, in Plasma Diagnostics, edited by W. Lochte-Holtgreven (North- Holland, Amsterdam, 1968) p.550.

2. A. W. DeSilva and G. C. Goldenbaum, in Methods of Experimental Physics, edited by H. R. Griem and R. Lovberg (Academic, New York, 1967), Sec. V9A.

3. D. E. Evans and J. Katzenstein, Rep. Pro. Phys. 32, $207-271$ (1969).

4. J. Sheffield, Plasma Scattering of Electromagnetic Radiation (Academic, New York, $1975)$

5. N. C. Luhman, Jr. and W. A. Peebles, in Laser Handbook, edited by M. Bass and M. L. Stitch (North-Holland, Amsterdam, 1985), Vol. 5, p. 455.

6. W. Rozmus, S. H. Glenzer, K. G. Estabrook, H. A. Baldis, and B. J. MacGowan, Modeling of Thomson scattering spectra in high-Z, laser-produced plasmas, Astrophys. J. S. in print.

7. E. M. Campbell et al., Laser Part. Beams 9, 209 (1991).

8. S. H. Glenzer, C. A. Back, K. G. Estabrook et al., Phys. Rev. Lett. 77, 1496 (1996).

9. S. II. Glenzer, W. Rozmus, B. J. MacGowan et al., Phys. Rev. Lett. 82, 97 (1999).

10. S. H. Glenzer, C. A. Back, L. J. Suter et al., Phys. Rev. Lett. 79, 1277 (1997).

11. S. H. Glenzer, T. L. Weiland, J. Bower, A. J. MacKinnon, and B. J. MacGowan, HighEnergy $4 \omega$ Probe Laser for laser-plasma experiments at Nova, Rev. Sci. Instrum. (1999), in press

12. G. B. Zimmerman and W. L. Kruer, Comments Plasma Phys. Controlled Fusion 2, 85 (1975).

13. L. J. Suter, R. L. Kauffman, C. B. Darrow et al., Phys. Rev. Lett. 73, 2328 (1994). 
Phys. Plasmas 3, 2057 (1996).

14. J. H. Nuckolls, Phys. Today 35, No. 9, 24 (1982).

15. J. D. Lindl, Phys. Plasmas 2, 3933 (1995).

16. S. W. Haan et al., Phys. Plasmas 2, 2480 (1995).

17. W. J. Krauser, Phys. Plasmas 3, 2084 (1996).

18. W. L. Kruer, The Physics of Laser Plasma Interactions (Addison-Wesley, New York, 1988).

19. T. P. Hughes, Plasmas and Laser Light (John Wiley and Sons, New York, 1975).

20. B. J. MacGowan, B. B. Afeyan, C. A. Back et al., Phys. Plasmas 3, 2029 (1996).

21. R. K. Kirkwood, B. B. Afeyan, C. A. Back et al., Phys. Rev. Lett. 77, 2706 (1996).

22. J. C. Fernandez, J. A. Cobble, B. H. Failor et al., Phys. Rev. Lett. 77, 2702 (1996).

23. S. H. Glenzer, L. J. Suter, R. E. Turner et al., Phys. Rev. Lett. 80, 2845 (1998).

24. D. G. Colombant and N. K. Winsor, Phys Rev. Lett. 38, 697 (1977).

25. S. I. Braginskii, in Reviews of Plasma Physics, ed. M. A. Leontovitch (Consultants Bureau, New York, 1965), Vol. 1, p. 205.

26. S. E. Bodner, Comments Plasma Phys. Controlled Fusion 6, 351 (1995).

27. J. A. Fejer, Can. J. Phys. 39, 716 (1961).

28. D. E. Evans, Plasma Phys. 12, 573 (1970).

29. E. Fünfer, B. Kronast, and H.-J. Kunze, Phys. Lett. 5, 125 (1963).

30. H.-J. Kunze, E. Fünfer, B. Kronast, and W. H. Kegel, Phys. Lett. 11, 42 (1964).

31. N. J. Peacock, D. C. Robinson, M. J. Forrest, P. D. Wilcock, and V. V. Sannikov, 
Nature (London) 224, 448 (1968).

32. H. Bindslev, Rev. Sci. Instrum. (1999), in press

33. H. A. Baldis, D. M. Villneuve, and C. J. Walsh, Can. J. Phys. 64, 961 (1986). H. A. Baldis, C. Labaune, E. Schifano, N. Renard, and A. Michard, Phys. Rev. Lett. 77, 2957 (1996).

34. J. E. Bernard, H. A. Baldis, D. M. Villeneuve, and K. G. Estabrook, Phys. Fluids 30, 3616 (1987).

35. M. D. Tracy, J. S. De Groot, K. G. Estabrook, and S. M. Cameron, Phys. Fluids B 4, 1576 (1992).

36. C. Labaune, H. A. Baldis, N. Renard et al., Phys. Rev. Lett. 75, 248 (1995). C. Labaune, H. A. Baldis, E. Schifano, et al., Phys. Rev. Lett. 76, 3727 (1996).

37. B. La Fontaine, H. A. Baldis, D. M. Villeneuve et al., Phys. Plasmas 1, 2329 (1994).

38. A. A. Offenberger, W. Blyth, A. E. Dangor, A. Djaoui, M. H. Key, Z. Najmudin, and J. S. Wark, Phys Rev. Lett. 71, 3983 (1993).

39. P. Young, Phys. Rev. Lett. 73, 1939 (1994).

40. M. J. Everett, A. Lal, D. Gordon, K. Wharton, C. E. Clayton, W. B. Mori, and C. Joshi, Phys. Rev. Lett. 74, 1355 (1995).

41. S. N. Dixit et al., Optics Lett. 21, 1715 (1996).

42. E. E. Salpeter, Phys. Rev. 120, 1528 (1960).

43. J. A. Fejer, Can. J. Phys. 38, 1114 (1960).

44. J. P. Dougherty and D. T. Farley, Proc. Roy. Soc. Ser. A 259, 79 (1960).

45. J. Renau, J. Geophys. Res. 65, 3631 (1960). 
46. Y. Q. Zhang, A. W. DeSilva, and A. N. Mostovych, Phys. Rev. Lett. 62, 1848 (1989). Y. Q. Zhang, and A. W. DeSilva, Phys. Rev. A 44, 3841 (1991).

47. J. F. Myatt et al., Phys. Rev. E 57, 3383 (1998).

48. J. Zheng, C. X. Yu, and Z. J. Zheng, Phys. Plasmas 4, 2736 (1997).

49. A. B. Langdon, Phys. Rev. Lett. 44, 575 (1980).

50. L. Spitzer, Jr. and R. Härm, Phys. Rev. 89, 977 (1953).

51. J. P. Matte, W. Rozmus, V. Yu. Bychenkov, and V. T. Tikhonchuk, Plasma Phys. Controlled Fusion 30, 1665 (1988). Phys. Rev. E 57, 3383 (1998).

52. G. B. Zimmerman and R. M. Moore, J. Quant. Spectrosc. and Radiat. Transfer 23, 517 (1980).

53. Y. T. Lee, J. Quant. Spectrosc. Radiat. Transfer 38, 131 (1987).

54. B. G. Wilson et al. in Radiative Properties of Hot Dense Matter, eds. W. Goldstein, C. Hooper, J. Gauthier, J. Seely, R. Lee (World Scientific, Singapore, 1991).

55. P. Young, private communication (1998).

56. E. A. Williams R. L. Berger, R. P. Drake et al., Phys. Plasmas 2, 129 (1995).

57. B. A. Hammel et al., Phys. Rev. Lett. 70, 1263 (1993).

58. M. D. Cable et al., Phys. Rev. Lett. 73, 2316 (1994).

59. A. A. Hauer et al., Phys. Plasmas 2, 2488 (1995).

60. N. D. Delamater et al., Phys. Plasmas 3, 2022 (1996).

61. V. Yu. Bychenkov, J. Myatt, W. Rozmus, and V. T. Tikhonchuk, Phys. Plasmas 1, 2419 (1994).

62. V. T. Tikhonchuk, W. Rozmus, V. Yu. Bychenkov, C. E. Capjack, E. Epperlein, Phys. 
Plasmas 2, 4169 (1995).

63. H. S. Rose, Phys. Plasmas 3, 1709 (1996). J. D. Moody et al., Phys. Rev. Lett. 77, 1294 (1996). D. S. Hinkel et al., Phys. Rev. Lett. 77, 1298 (1996).

64. J. R. Albritton, E. A. Williams, I. B. Bernstein, and K. P. Swartz, Phys. Rev. Letters 57, 1887 (1986). J. F. Luciani, P. Mora, and R. Pellat, Phys. Fluids 28, 835 (1985). A. R. Bell, R. G. Evans, and D. J. Nicholas, Phys. Rev. Lett. 46, 243 (1981).

65. J. A. Stamper, K. Papadopoulos, R. N. Sudan, E. A. McLean, and J. M. Dason, Phys. Rev. Lett. 26, 1012 (1971).

66. M. G. Haines, Phys. Rev. Lett. 78, 254 (1997).

67. D. A Tidman and R. A. Shanny, Phys. Fluids 17, 1207 (1973).

68. M. G. Haines, Can. J. Phys. 64, 912 (1986).

69. W. Seka, B. B. Afeyan, R. Boni, L. M. Goldman, R. W. Short, K Tanaka, and T. W. Johnston, Phys. Fluids 28, 2570 (1985).

70. S. H. Glenzer, F. B. Rosmej, R. W. Lee, C. A. Back, K. G. Estabrook, B. J. MacGowan, T. D. Shepard, and R. E. Turner, Phys. Rev. Lett. 81, 365 (1998).

71. K. G. H. Baldwin, J. P. Marangos, and D. D. Burgess, Plasma Phys. and Controlled Fusion 30, 1141 (1988).

72. H.-J. Wesseling and B. Kronast, J. Phys. D 29, 1035 (1996); Europhys. Lett. 33, 273 (1996).

73. L. Kellerer, Z. Physik 232, 415 (1970), ibid, 239, 147 (1970). 


\section{FIGURES}

Fig. 1. Schematic of the open-geometry disk experiments.

Fig. 2. Experimental $2 \omega$ TS spectra measured while the heater beam is on at $t=1.4$ ns showing the ion and the electron feature alon with theoretical fits. The dashed curves are calculations without gradients.

Fig. 3. Averaged charge number, $\bar{Z}$, electron temperature, $T_{e}$, and electron density, $n_{e}$, as a function of time measured with TS. Calculations with DCA are in agreement with the observations while calculations with XSN are not consistent with the charge number and density data in the recombination phase of the plasma.

Fig. 4. Experimental TS data from a two-ion species plasma as a function of time (a). The spectrum shows two ion acoustic waves due to $A u$ and $B e$ ions (b). The variations of the ratio $T_{e} / T_{i}$ indicates the sensitivity of the relative intensity of the peaks to the ion temperature (c).

Fig. 5. Schematic of the $4 \omega$ TS experiments in Nova scale-1 hohlraums.

Fig. 6. Time-resolved TS data from a $\mathrm{CH}_{4}$-filled hohlraum heated with 10 smoothed beams (a). Examples of fits to the spectra at $t=1.2 \mathrm{~ns}$ and $t=1.6 \mathrm{~ns}$ giving the electron and ion temperature of the plasma (b).

Fig. 7. Time-resolved two-dimensional x-ray images of the hohlraum gold emission with energies $E>2.5 \mathrm{keV}$ observed along the hohlraum axis. Results are shown from empty hohlraums (a) and methane-filled $\left(\mathrm{CH}_{4}\right)$ hohlraums (b). The diameter of the hohlraums is $1,600 \mu \mathrm{m}$ and that of the LEH through which the observations have been made is $1,200 \mu \mathrm{m}$. 
Fig. 8. Electron temperatures measured on the axis of a methane-filled hohlraum at various distances from the hohlraum center along with the results from our radiation-hydrodynamic modeling. The calculations with magnetic fields clearly show a temperature gradient in agreement with the experimental data while the calculations with standard heat transport inhibition $f=0.05$ result in a rather isothermal plasma.

Fig. 9. Calculated magnetic field contours for methane-filled hohlraums at various times during the heating of the hohlraum. The fields inhibit thermal transport in plasma regions through which the heater beams (indicated as dashed lines) propagate on their way to the hohlraum walls.

Fig. 10. Calculated electron temperature contours for methane-filled hohlraums at $t=1.3 \mathrm{~ns}$. The magnetic field causes the development of hot regions in the hohlraum through which the heater beams (indicated as dashed lines) propagate.

Fig. 11. Axial ion and electron temperatures as a function of time at a distance of $800 \mu \mathrm{m}$ from the center of a methane-filled hohlraum. The calculations are seen to be in good agreement with the experimental data. The fast rise of $T_{i}$ at $t=1.2 \mathrm{~ns}$ is due to stagnation of the plasma on the axis of the hohlraum.

Fig. 12. Time-resolved TS data from a $\mathrm{C}_{3} \mathrm{H}_{8}$-filled hohlraum heated with 10 smoothed beams (a). Also shown are the ion and electron temperatures as a function of time at a distance of $800 \mu \mathrm{m}$ from the hohlraum center (b). The stagnation of the plasma on the axis of the hohlraum indicated by the fast rise of $T_{i}$ occurs at $t=1.5 \mathrm{~ns}$ which is delayed by $0.3 \mathrm{~ns}$ compared to $\mathrm{CH}_{4}$-filled hohlraums. 


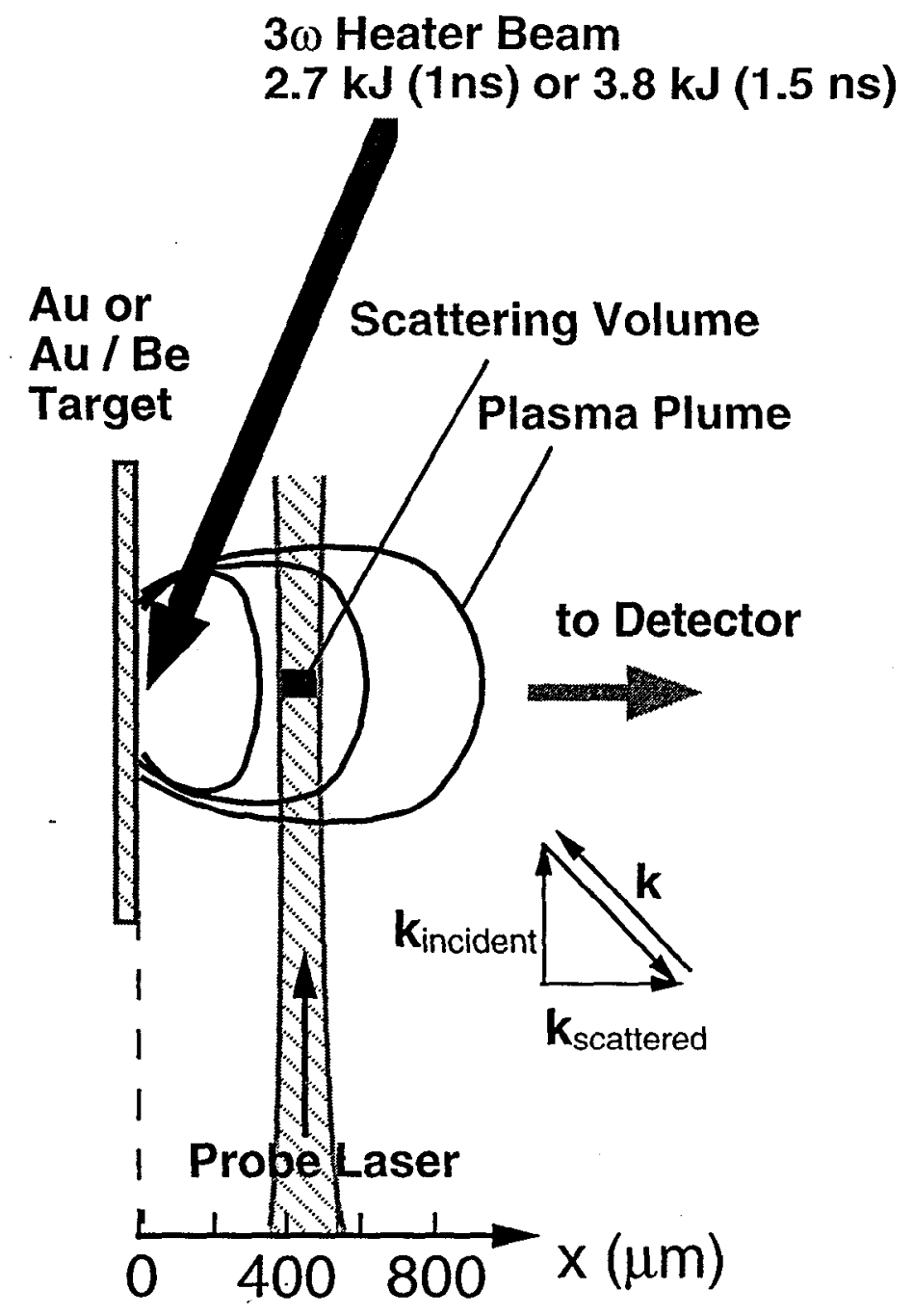

Figure 1 


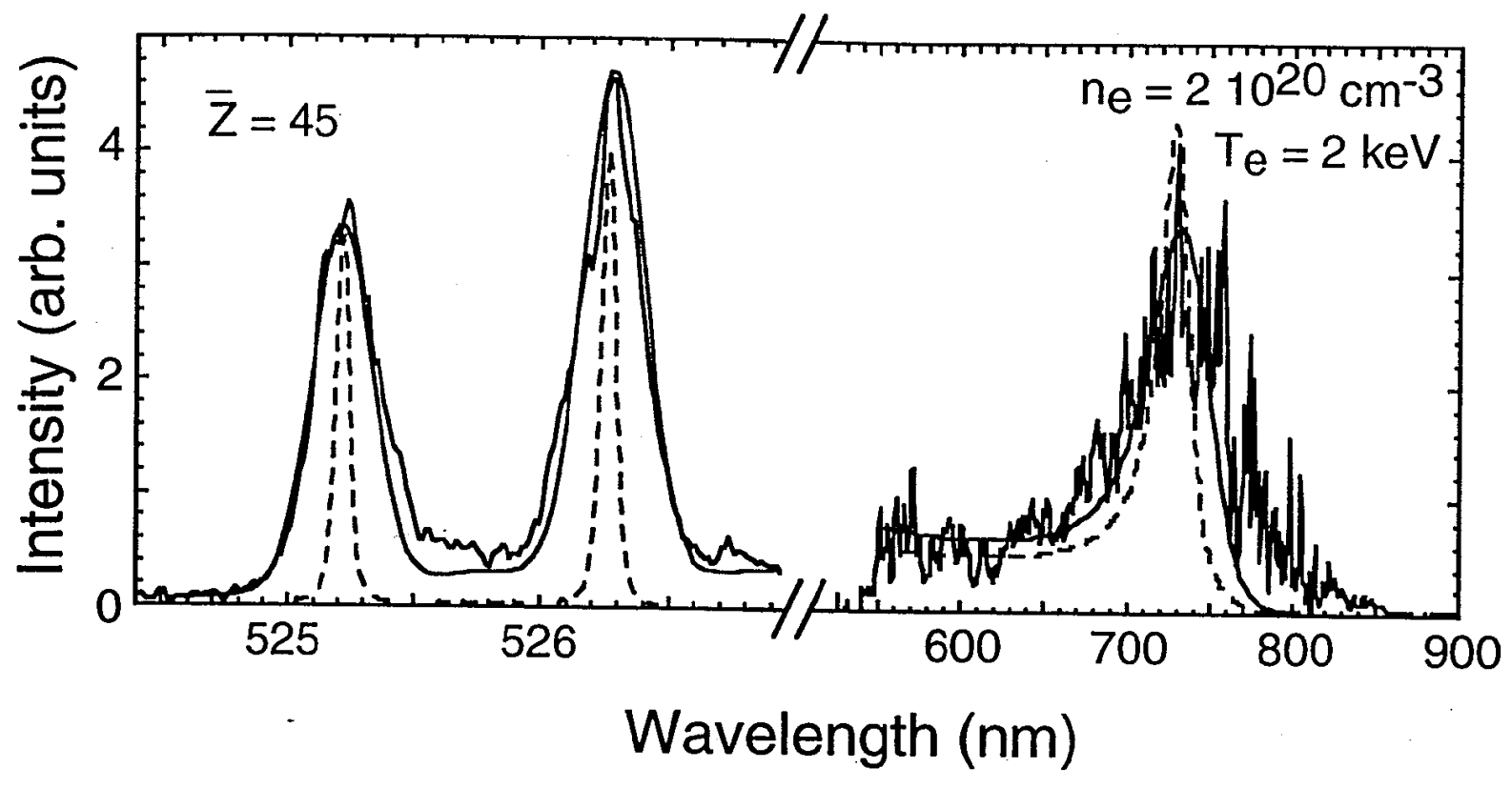

Figure 2 


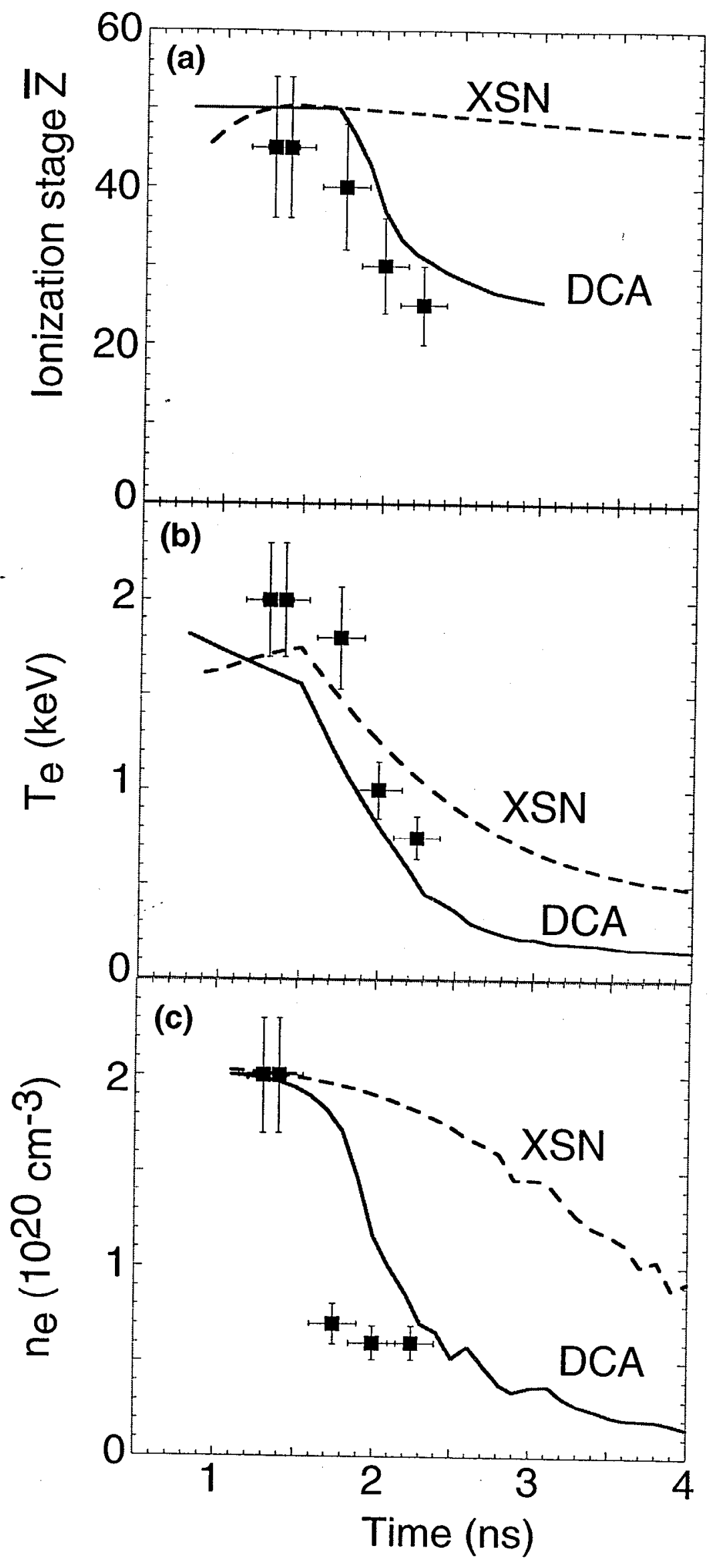

Figure 3 

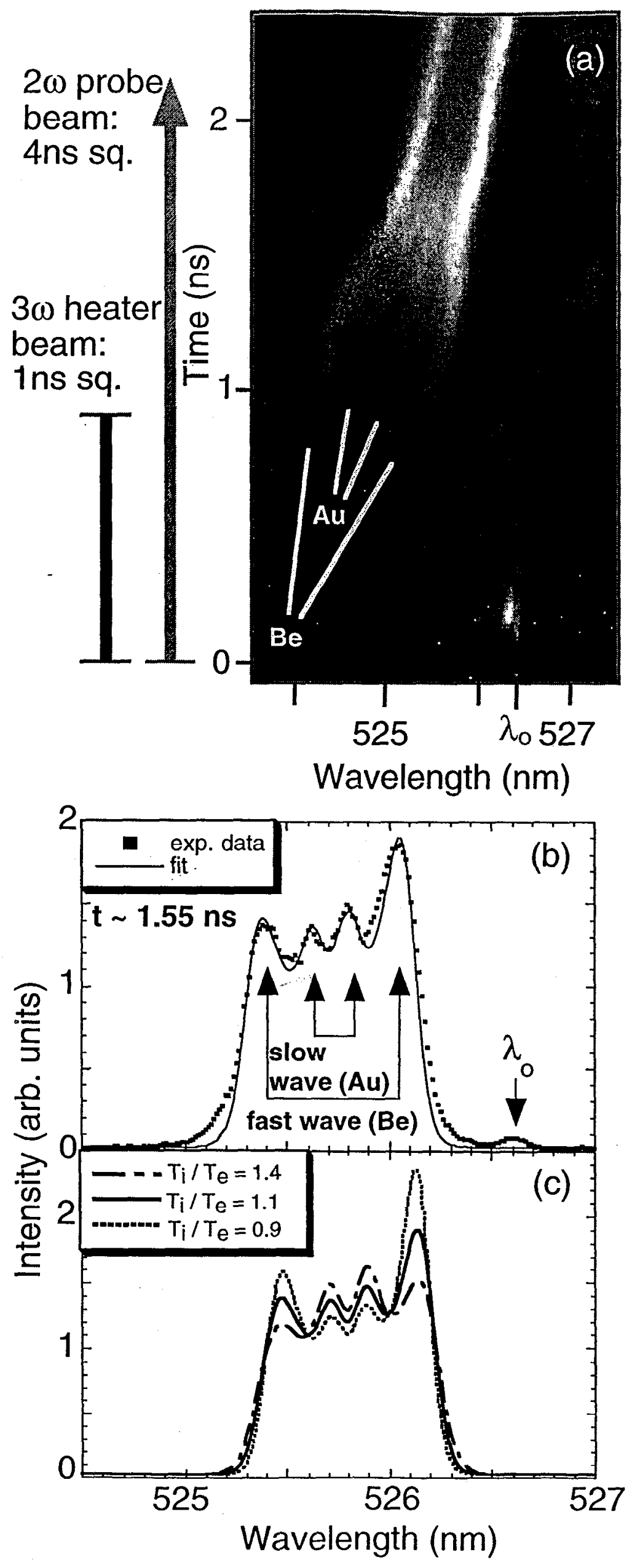


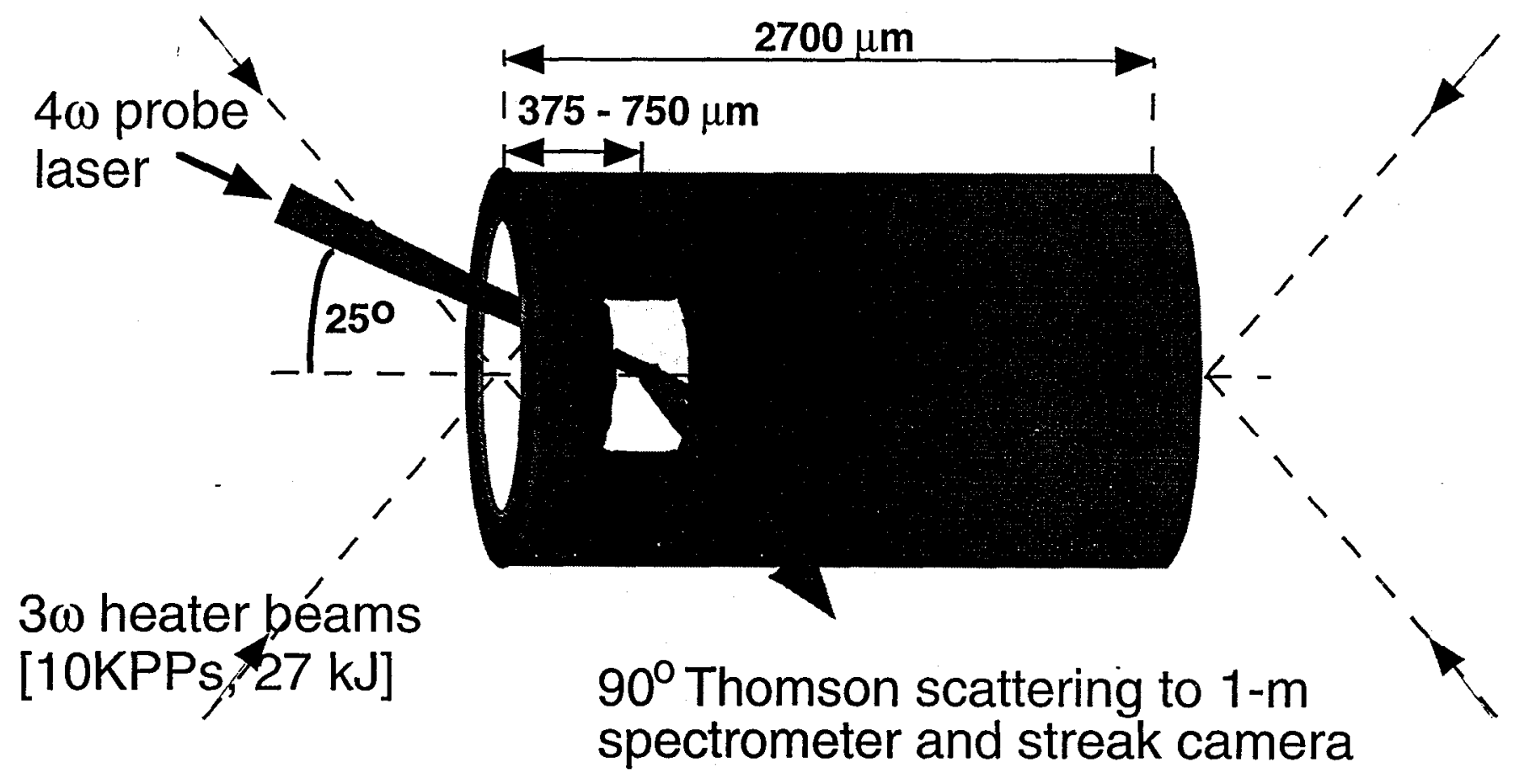

Figure 5 


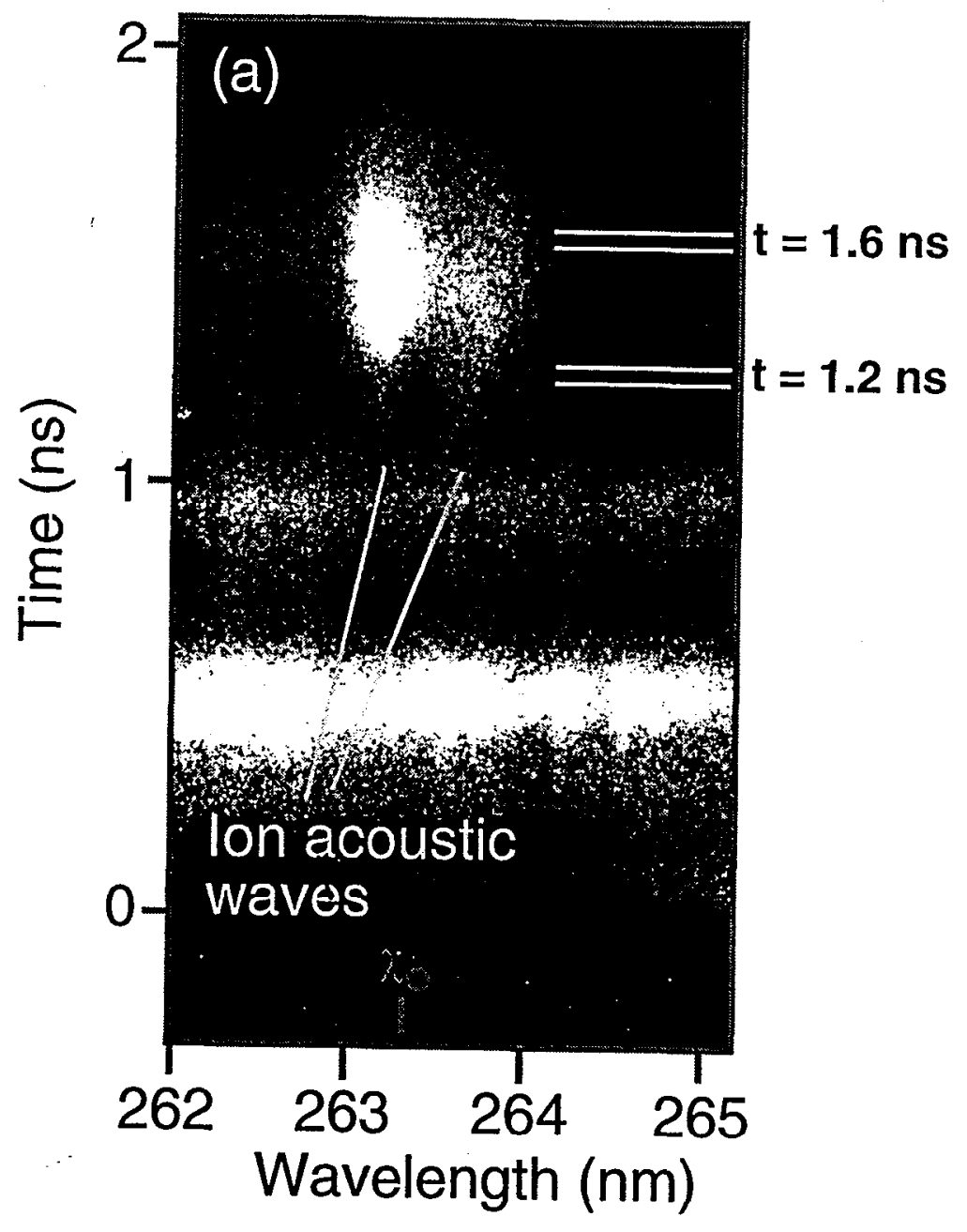

Figure 6 (a) 


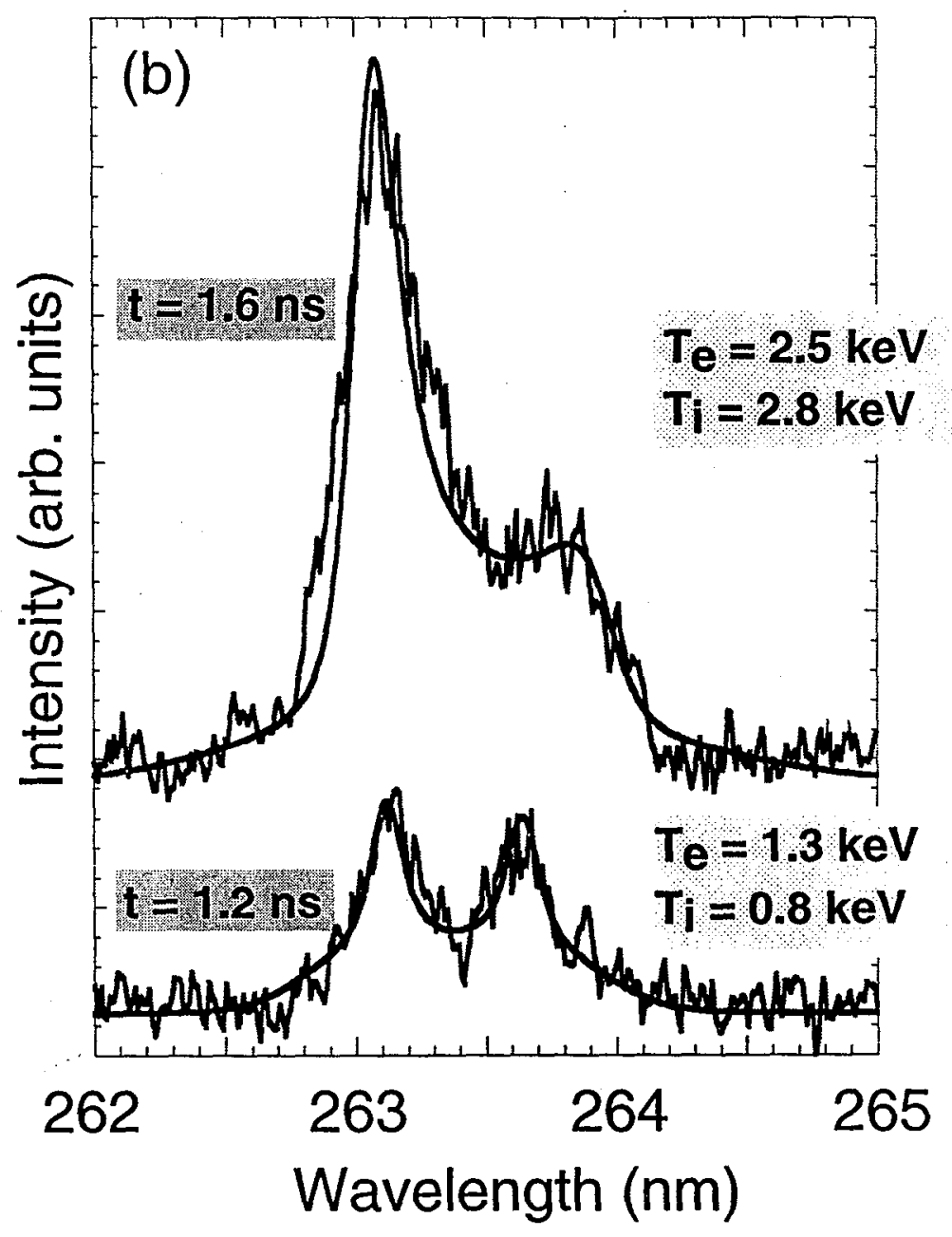

Figure 6 (b) 
(a) Empty Hohlraum
$t=t_{0}+0.2 n s$
$\mathrm{t}=\mathrm{t}_{0}+0.3 \mathrm{~ns}$
$t=t_{0}+0.45$ ns
$t=t_{0}+1.3$ ns
$t=t_{0}+1.8 \mathrm{~ns}$
\%
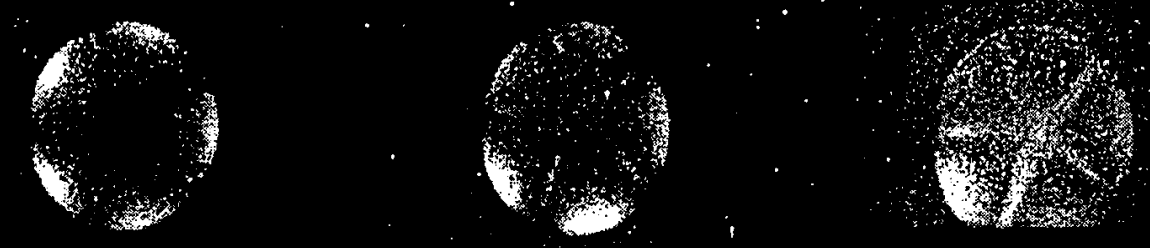

(b) $\mathrm{CH}_{4}$-filled Hohlraum

$$
t=t_{0}+0.2 n s
$$$$
t=t_{0}+0.3 n s
$$$$
t=t_{0}+0.45 \mathrm{~ns}
$$$$
t=t_{0}+1.3 n s
$$$$
t=t_{0}+1.8 \mathrm{~ns}
$$
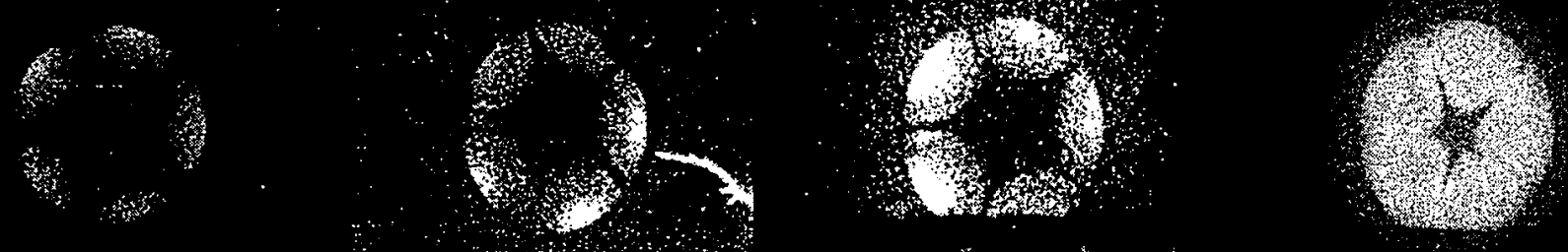

Figure 7 


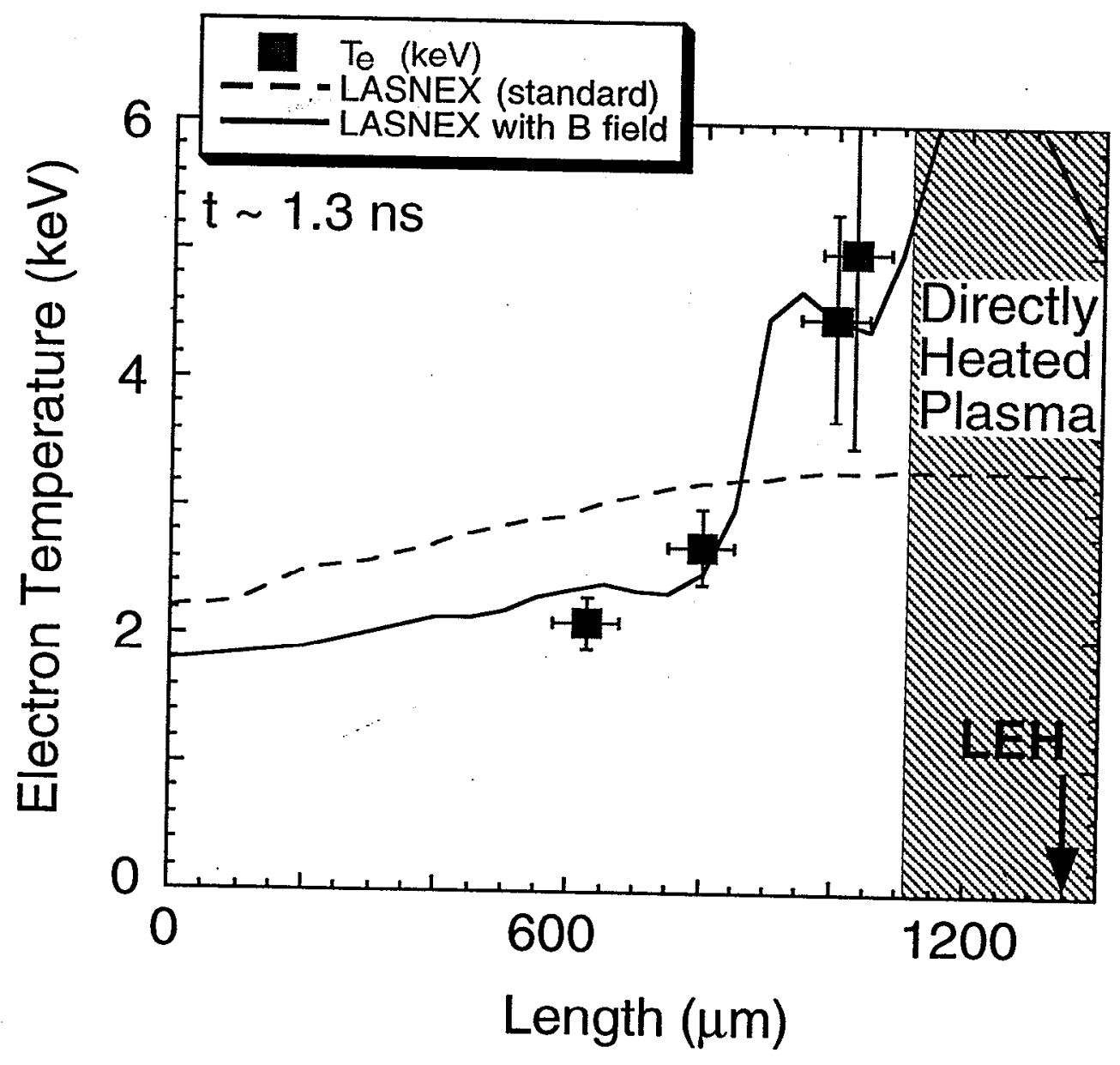

Figure 8 


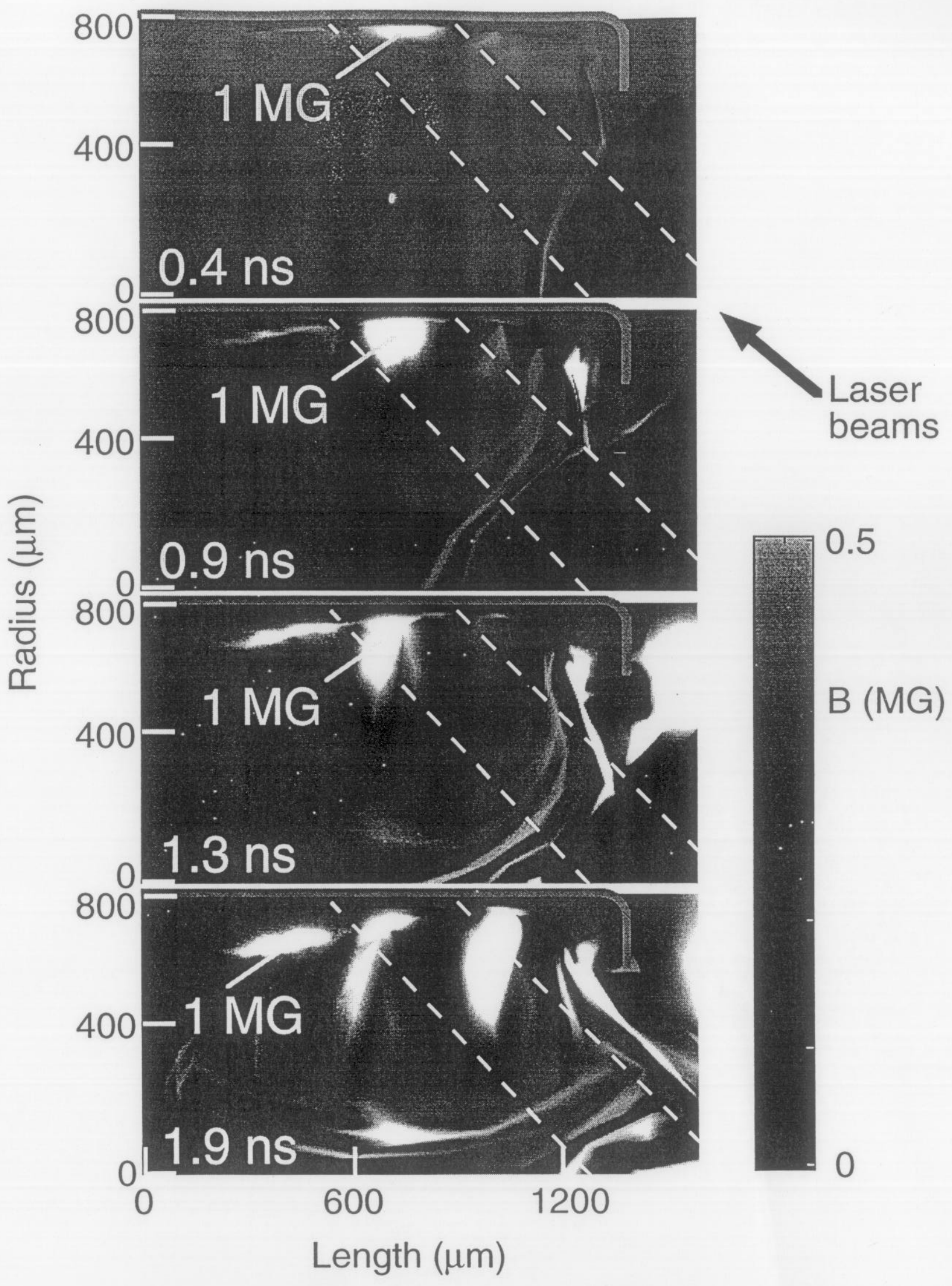

Figure 9 


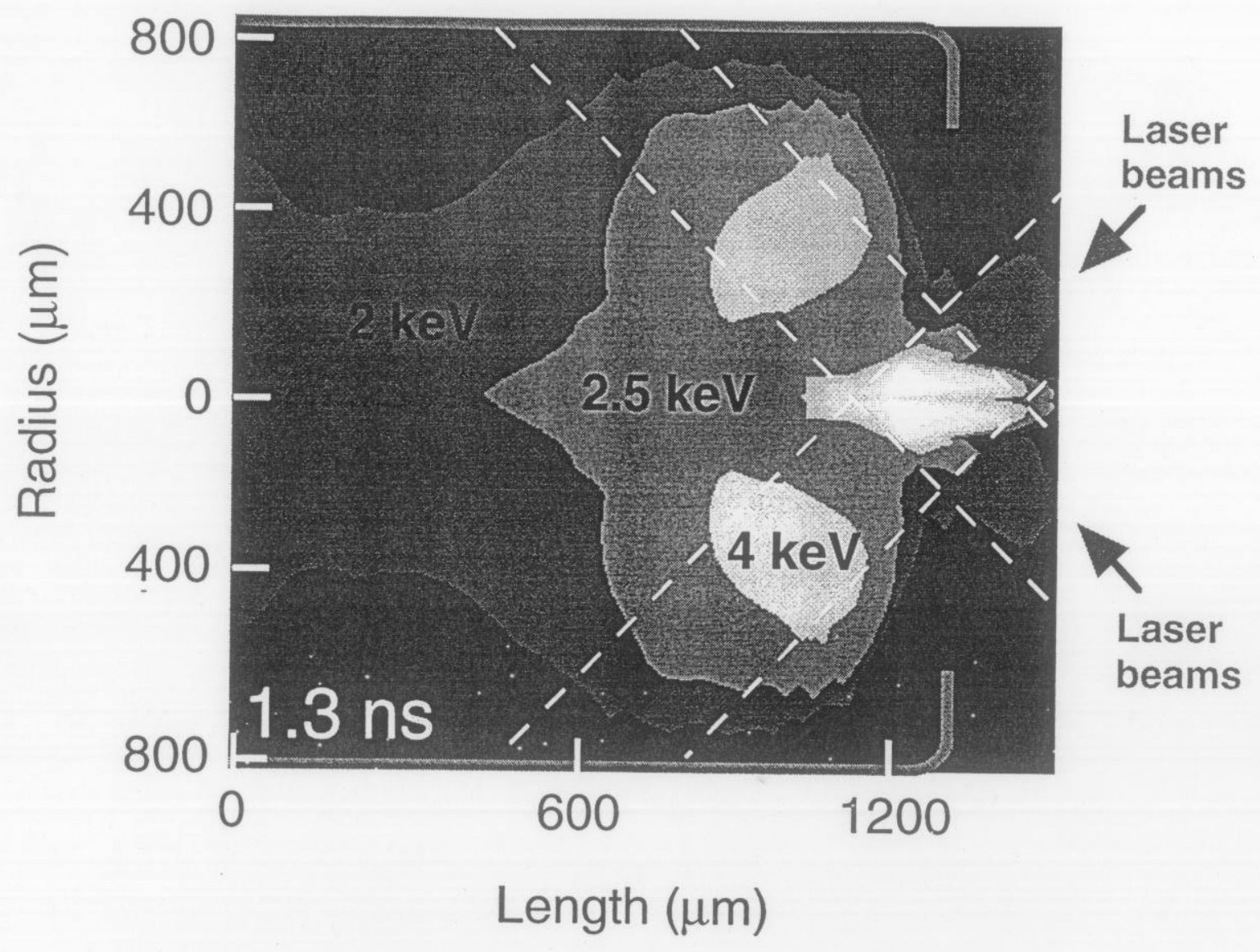

Figure 10 


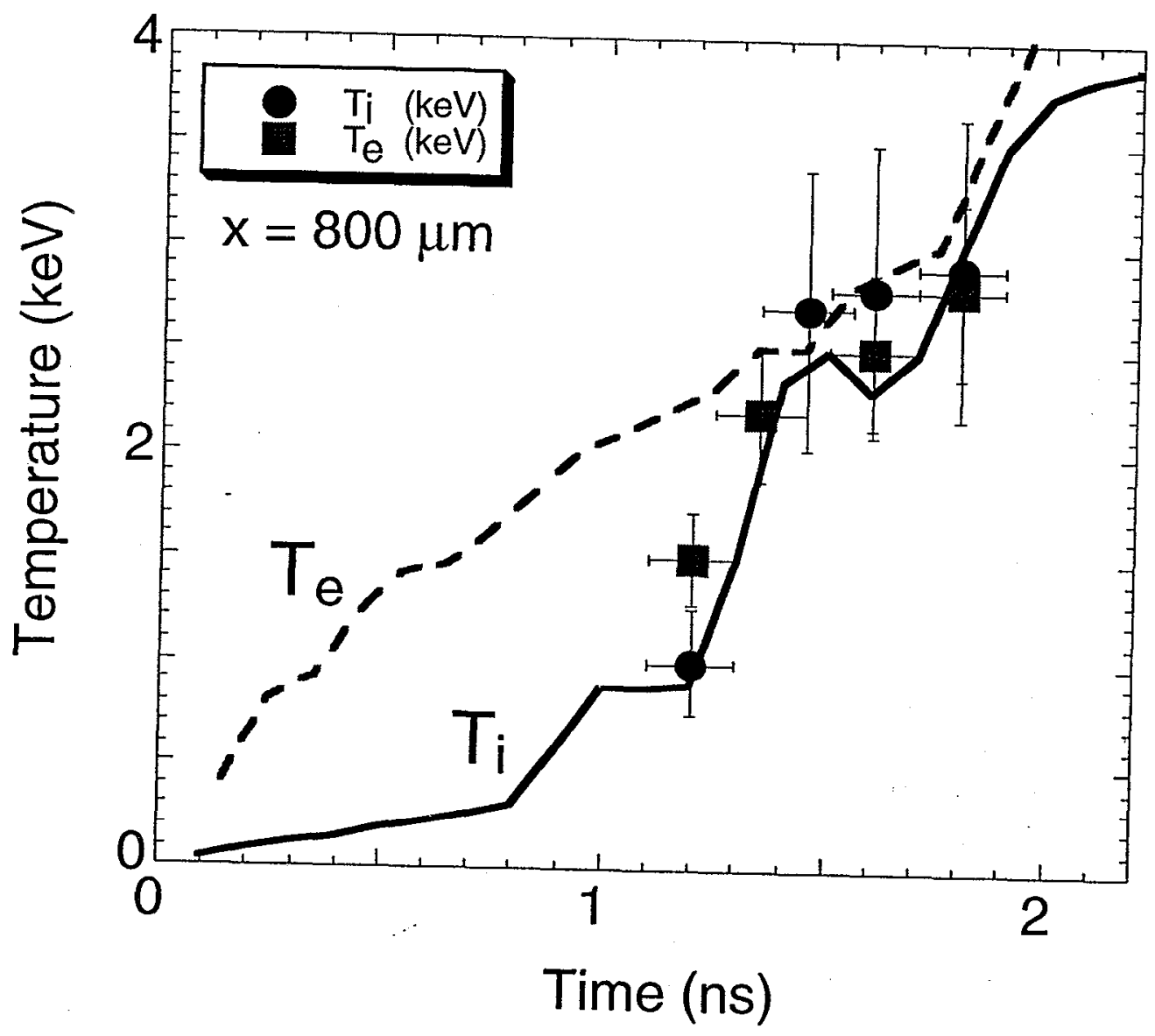

Figure 11 


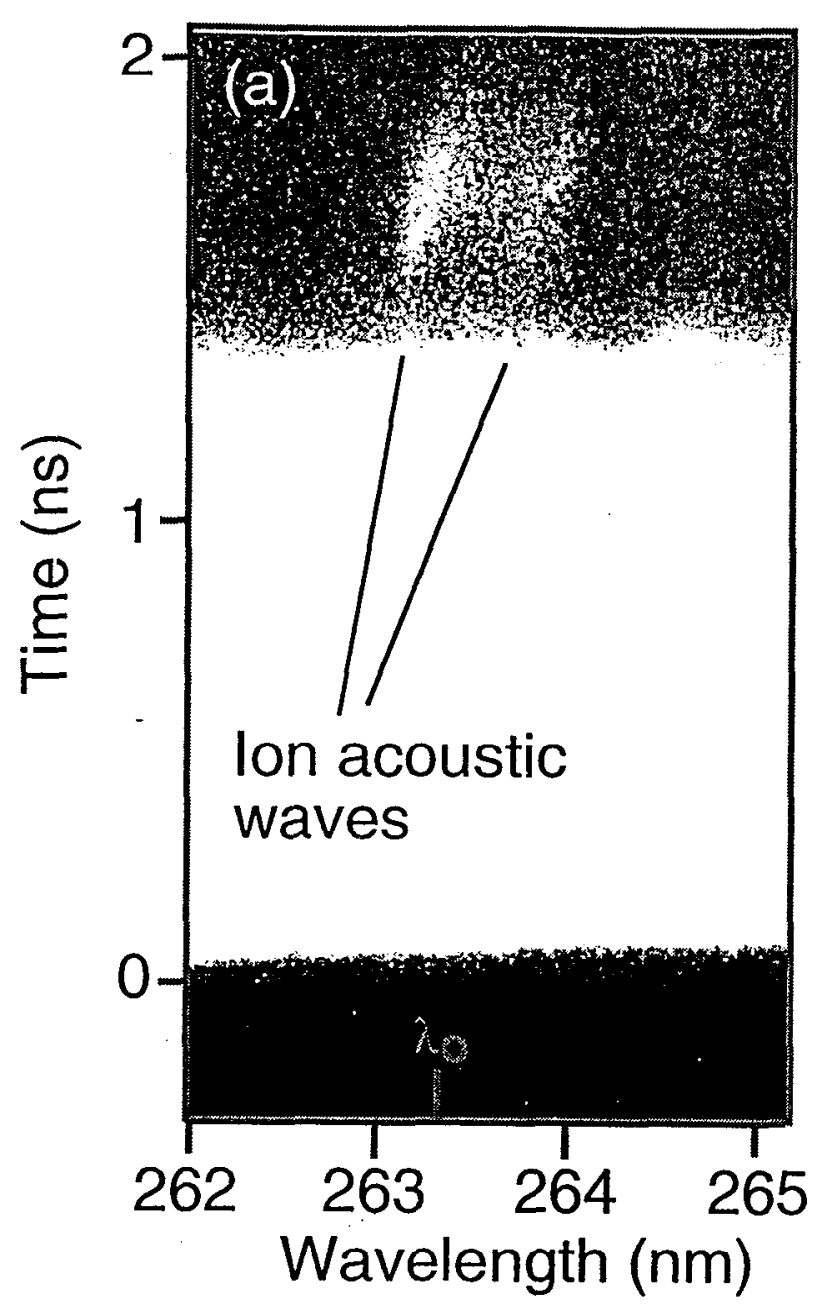

Figure 12(a) 


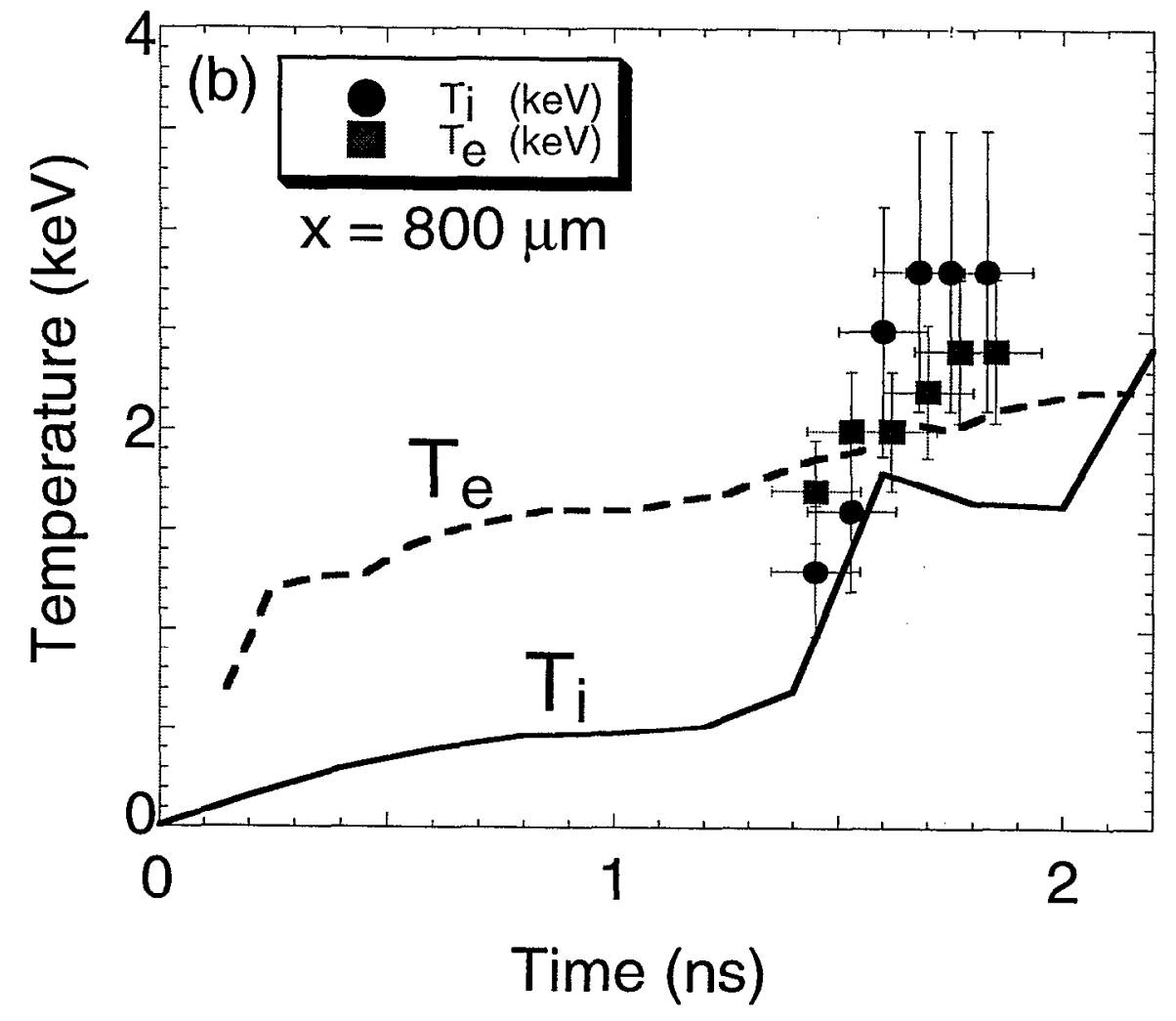

Figure 12(b) 